\title{
Transfer Path Analysis and Contribution Evaluation Using SVD- and PCA-Based Operational Transfer Path Analysis
}

\author{
Wei Cheng $\mathbb{D}^{1,2}{ }^{1,2}$ Diane Blamaud, ${ }^{2,3}$ Yapeng Chu, ${ }^{1,2}$ Lei Meng, ${ }^{4}$ Jingbai Lu, ${ }^{1,2}$ \\ and Wajid Ali Basit ${ }^{1,2}$ \\ ${ }^{1}$ State Key Laboratory for Manufacturing Systems Engineering, Xi'an Jiaotong University, Xi'an 710049, Shaanxi, China \\ ${ }^{2}$ School of Mechanical Engineering, Xi'an Jiaotong University, Xi'an 710049, Shaanxi, China \\ ${ }^{3}$ Arts et Métiers Paris Tech, Paris 75000, France \\ ${ }^{4}$ School of Automation, Wuhan University of Technology, Wuhan 430070, Hubei, China
}

Correspondence should be addressed to Wei Cheng; chengw@xjtu.edu.cn

Received 29 January 2020; Revised 15 June 2020; Accepted 3 July 2020; Published 30 July 2020

Academic Editor: Luca Landi

Copyright ( $\odot 2020$ Wei Cheng et al. This is an open access article distributed under the Creative Commons Attribution License, which permits unrestricted use, distribution, and reproduction in any medium, provided the original work is properly cited.

To quantitatively identify the transfer paths and evaluate path contributions of shell structures, an singular value decomposition(SVD-) and principal component analysis- (PCA-) based operational transfer path analysis method is constructed and studied in this paper. Firstly, SVD is used to determine the contribution of each path and reduce crosstalk. Secondly, PCA is applied to reduce the influence of unwanted frequency components and thus reduce noises. This allows the presented OTPA to be more accurate than its traditional counterpart. Once the transmissibility function is obtained, the response synthesis is determined, and the transfer path analysis and path contribution evaluation can be effectively carried out. Numerical and experimental case studies are carried out to validate and test the performance of the presented method. Furthermore, a comprehensive observing the influences of correlation between sources and distance of sources and receiver is also provided. Generally, this paper provides accurate transfer path analysis and path contributions for mechanical systems, which can benefit vibration and noise monitoring and reduction through vibration reduction structure design for new equipment or vibration damping on the major vibration transfer paths for current equipment.

\section{Introduction}

Over the past decades, the demand for comfortable, silent, and stealthy technologies has extensively grown. The knowledge of transmission and distribution of vibration and noise signals, being the major concern while dealing with noise and vibration and harshness (NVH) testing and the vibroacoustic source prediction, is acquired using transfer paths concept [1-5]. In this prospect, vibration and noise reduction techniques for cars, heavy duty vehicles, and high-speed trains are also shinning research hotspot $[6,7]$. Transfer path analysis is often used to analyze the transfer characteristics of vibration and noise signals for a mechanical system. Generally, the source of vibrations and noises refers to actively vibrating components (such as engines, gearing systems, or turbochargers). For the purpose of solving the noise problem caused by vibration, TPA studies the energy flow from the source through a series of mechanical structures or air to a specified receiving point, which is known as path. Furthermore, operational transfer path analysis (OTPA) has been attracting a lot of attention for its rapidity and simplicity in modeling complex mechanical systems. However, because of noise and crosstalk, the traditional OTPA presents consequent errors in response synthesis evaluation and misjudgments in the transfer function determination. Therefore, crosstalk cancellation and improving the accuracy of OTPA prove to be of great research significance, which can benefit vibration and noise monitoring, reduction and control through vibration reduction structure design, or vibration damping on the major vibration transfer paths. 
OTPA has been widely used in engineering for its rapidity and convenience [8,9]. Deklerk and Ossipov [10] applied OTPA based on singular value decomposition (SVD) to analyze tire noises. Yan and Weikang [11] studied influences of radiation noises of plates on interior noises of a car by OTPA based on local singular value decomposition. Reising et al. [12] applied OTPA based on SVD to effectively assess acoustic characteristics of the driven axle in automobiles. Commonly, the traditional OTPA identifies transmissibility functions to analyze the transfer path characteristics [6]. However, for mechanical systems with complex structures, it is difficult to clearly distinguish the vibration transmission path, which can cause path misjudgments and errors in the response synthesis [10, 13]. To improve the accuracy of OTPA based on SVD, Roozen and Leclère [14] estimated transmissibility functions based on the responses generated by hammer strokes instead of operating conditions. Generally, the noise and crosstalk problem is the root cause of inaccuracy for the traditional OTPA and errors remain. SVD and principal component analysis (PCA) have been successfully used by Toome in his study of a source contribution prediction at low frequency [15] and by Ström in the OTPA of a high-speed train bogie [16]. However, the crosstalk or noise problem for the traditional OTPA is yet to be solved in a satisfying way, and until now, little literature is found to solve the crosstalk problem of OTPA by SVD and PCA.

To quantitatively identify the transfer paths and evaluate path contributions of shell structures, an SVD- and PCAbased operational transfer path analysis method is constructed and studied in this paper. Firstly, SVD is used to reduce the influence of environmental noise upon data processing and effectively improve the signal-to-noise ratio (SNR), and then, the PCA is applied to reduce the influence of unwanted frequency components and thus reduce the crosstalk. This allows the novel OTPA to be more accurate than its traditional counterpart. Once the transmissibility function is obtained, the response synthesis is determined and the transfer path analysis can be effectively carried out. Thus, the crosstalk problem of the traditional OTPA is remarkably improved.

The remainder of this paper is organized as follows: Section 2 introduces the fundamental theory of SVD, PCA, and the proposed method; Section 3 provides numerical case studies of spherically radiating sources and comparatively studies the accuracies of the proposed OTPA method and the traditional OTPA method; Section 4 gives an experimental study on a cylindrical steel shell structure and further validates the effectiveness of the proposed method; Section 5 provides a comprehensive study of the proposed method and examines the parameter effects; and finally, the conclusions of this work are summarized in Section 6.

\section{Theory of PCA- and SVD-Based OTPA}

2.1. Traditional Model of OTPA. The OTPA method is based on the knowledge of inputs and outputs of the system. The signal can be processed using crosstalk cancellation (CTC), SVD, and PCA to establish the linearized transfer function
(TF) matrix between sets of inputs and outputs as first established by Noumura and Yoshida [17]. When using the model of TPA, inescapable frequency response function testing and structural load identification are time-consuming, which drives us turn to more convenient method OTPA. In OTPA, reference points are added at the position close to each excitation source at the passive side. As a result, the structural load in TPA is replaced by the signal of reference points, while frequency response function is replaced by the transfer rate function matrix from the reference point to the target point. Different from the TPA method, the input signal of OTPA is measured at a reference point, and the output signal is measured at a target point.

Usually, the physical quantities measured are motions (displacement, velocity, or acceleration), forces, and sound pressures. In the automotive industry, accelerometers or displacement sensors are used to measure the vibration signals, and sound pressure sensors are used for sound levels during noise measurements. For operational transfer path analysis, transfer properties of simple linear systems can be described as

$$
\mathbf{Y}=\mathbf{H X}
$$

where $Y$ is the response matrix of size $n, X$ is the input excitation matrix of size $m$, and $H$ is the transfer function matrix. More minutely, it can be expressed as

$$
\left[\begin{array}{c}
\mathbf{y}^{(1)} \\
\vdots \\
\mathbf{y}^{(n)}
\end{array}\right]=\left[\begin{array}{ccc}
\mathbf{H}_{11} & \cdots & \mathbf{H}_{1 m} \\
\vdots & \ddots & \vdots \\
\mathbf{H}_{n 1} & \cdots & \mathbf{H}_{n m}
\end{array}\right]\left[\begin{array}{c}
\mathbf{x}^{(1)} \\
\vdots \\
\mathbf{x}^{(m)}
\end{array}\right]
$$

where $m$ and $n$ are the number of measuring points for all inputs (reference) and outputs (response).

In practical operational measurements for $\mathrm{NVH}$ analysis, input and output measurements change rapidly, and hence, different transfer functions are defined to interlink these output changes with varying inputs; this relation for different conditions can be described as an expanded form of equation (2):

$$
\left[\begin{array}{ccc}
\mathbf{y}_{1}^{(1)} & \cdots & \mathbf{y}_{r}^{(1)} \\
\vdots & \ddots & \vdots \\
\mathbf{y}_{1}^{(n)} & \cdots & \mathbf{y}_{r}^{(n)}
\end{array}\right]=\left[\begin{array}{ccc}
\mathbf{H}_{11} & \cdots & \mathbf{H}_{1 m} \\
\vdots & \ddots & \vdots \\
\mathbf{H}_{n 1} & \cdots & \mathbf{H}_{n m}
\end{array}\right]\left[\begin{array}{ccc}
\mathbf{x}_{1}^{(1)} & \cdots & \mathbf{x}_{r}^{(1)} \\
\vdots & \ddots & \vdots \\
\mathbf{x}_{1}^{(m)} & \cdots & \mathbf{x}_{r}^{(m)}
\end{array}\right],
$$

where $r$ is the number of operating conditions during experiments. The relations between the inputs and outputs remain linear even the content changes with each configuration.

To simplify and make the problem invertible, an equal number of blocks (operating configurations) and measurement points $(m=r)$ are considered. Thus, the transfer function can be obtained by simply inverting $\mathbf{X}$ :

$$
\mathbf{H}=\mathbf{Y X}^{-1} \text {. }
$$

However, this method is not commonly used; as in most cases in the industry, the input matrix is not always square $(m \neq n)$. The problem can be seen as a least-square 
optimization problem, which is required to choose a number of measurement blocks more than the number of input measurements $(r>m)$ for the equation to be solvable.

When the number of measurement blocks is greater than the number of measurement points, the traditional method used is the least-square solving method, which introduces a residue $\mu$ to the equation (1), which then becomes

$$
\mathbf{Y}=\mathbf{H} \mathbf{X}+\mu \text {. }
$$

with

$$
\mathbf{H}=\mathbf{Y X}^{+} \text {, }
$$

$\mathbf{X}^{+}$being the pseudoinverse of $\mathbf{X}$ and where $\mathbf{X}^{+}$is defined as

$$
\mathbf{X}^{+} \triangleq\left(\mathbf{X}^{T} \mathbf{X}\right)^{-1} \mathbf{X}^{T} .
$$

The residue $\mu$ can be determined by the following equation:

$$
\mu=\left(1-\mathbf{X}\left(\mathbf{X}^{T} \mathbf{X}\right)^{-1} \mathbf{X}^{T}\right) \mathbf{Y} .
$$

The first implemented method to solve OTPA problems shows potential errors. Indeed, the noise is drastically amplified during the pseudoinverse calculation $\left(X^{T} X\right)^{-1}$ when the input sources are highly correlated. Since measurement noise is impossible to avoid in real life cases, this method needs to be improved with SVD to prevent poor estimates of the transfer function in the determination of the input matrix pseudoinverse. The next step is to lower the noise level and crosstalk between measurement channels. Thus, the crosstalk cancellation method is often preferred. Another problem with the least-square method is that it uses pseudoinverse, which uses a discontinuous mapping of the data when the matrix is not full rank. In other words, the pseudoinverse of a rank deficient matrix is sensitive to noisy data and needs regularization.

Furthermore, as stated before, one of the major causes of error in TPA and more precisely in OTPA is due to numerical errors, which come from the fact that correlation between inputs is not taken into account when the transfer matrix is calculated as shown in equation (6). There is a part of the source contribution that is not its own but due to other sources, which cannot be identified by the captors themselves, and have to be sorted out through signal analysis. Generally, there are several ways that can remove those parasite contributions to the signal, such as SVD or crosstalk cancellation (CTC).

\subsection{Theory of SVD and PCA Improved OTPA}

2.2.1. SVD Method. For denoising and linear regression analysis of the complex data set, all the singular values (or eigenvalues) of the data set are determined. SVD is commonly used to find the correlation between components of a data set to undo the correlation between them by making each value orthogonal (uncorrelated) to the other singular values. The strongest correlated component of the data set is considered as the first singular value. The remaining of the data set is then run through SVD again to find the next strongest singular value, until the full data set is defined. The mathematical expression of SVD is

$$
\mathbf{X}=\mathbf{U} \Sigma \mathbf{V}^{T} \text {, }
$$

where $\mathbf{X}$ is the matrix of input measurements of dimension $r \times m, \mathbf{U}$ is the unitary column-orthogonal matrix of dimension $r \times m, \boldsymbol{\Sigma}$ is the singular value matrix of dimension $m \times m$, and $\mathbf{V}^{T}$ is the transpose of an unitary column-orthogonal matrix $\mathbf{V}$ of dimension $m \times m$.

In order to find all the component matrices satisfying equation (9), the following relations are used:

$$
\begin{aligned}
& \mathbf{X}^{T} \mathbf{X}=\mathbf{V} \boldsymbol{\Sigma}^{T} \mathbf{U}^{T} \mathbf{U} \boldsymbol{\Sigma} \mathbf{V}^{T}=\mathbf{V}\left(\boldsymbol{\Sigma}^{T} \boldsymbol{\Sigma}\right) \mathbf{V}^{T}, \\
& \mathbf{X} \mathbf{X}^{T}=\mathbf{U} \boldsymbol{\Sigma} \mathbf{V}^{T} \mathbf{V} \boldsymbol{\Sigma}^{T} \mathbf{U}^{T}=\mathbf{U}\left(\boldsymbol{\Sigma} \boldsymbol{\Sigma}^{T}\right) \mathbf{U}^{T} .
\end{aligned}
$$

The expression of the pseudoinverse matrix $\mathbf{X}^{+}$is obtained through the SVD of $\mathbf{X}$, as shown in the previous equation:

$$
\mathbf{X}^{+}=\mathbf{V} \boldsymbol{\Sigma}^{-1} \mathbf{U}^{T} .
$$

Using the previously stated equations, an estimate of the transfer path matrix can be found, $\mathbf{H}$, with the following expression:

$$
\widetilde{\mathbf{H}}=\mathbf{Y} \mathbf{V} \boldsymbol{\Sigma}^{-1} \mathbf{U}^{T} .
$$

This equation is an estimate of solution of the transfer path matrix. However, the presence of crosstalk in the data still needs to be addressed. To do so, PCA technique is implemented.

2.2.2. PCA Method. PCA is used to eliminate the noises in overall response and focuses on only meaningful information and to be able to compute the transfer function without problem. Its principle is close to that of the contribution rate. PCA is a method employed to reduce a data set made of correlated variables to a smaller data set which holds most of the original information. In other words, PCA is an orthogonal transformation to remove correlation between data sets.

The variables obtained after the orthogonal transformation are called principal components (PCs), which are ranked according to their variances within the data set. Thus, the PC with the largest variance will be the first PC and so on until the PCs show very little variance and are almost constant. For example, when conducting PCA on vibration data, the strongest contribution is due to bending, then that would be the first PC. The PCA would continue the remaining data (i.e., the residual), and the next strongest contribution, for example, torsional waves, would be considered the second PC. The analysis would continue in this manner for all vibration DOFs and is conducted similarly for sound pressure, with the ranking of PCs related to the difference phases between PC and data set.

In operational transfer path analysis with PCA technique, whole data set is converted into the frequency domain and the DFT spectra show the variables (input data) for each measuring point. Furthermore, this PCA technique is 
conducted for all experimental observations $r$ that are considered as a part of excitation matrix $X$. Consequently, the OTPA using PCA determines the extent of common signal sharing (correlation) of all measurement points.

The PC contribution rates are obtained by multiplying $\Sigma$ by the eigenvector $\mathbf{U}$, and crosstalk is then taken off the problem after being identified as the smallest PCs, which is done through dividing the contribution scores by the total sum of principal component scores. After obtaining the percentage of each $\mathrm{PC}$, the crosstalk can be determined. The crosstalk cancelled, and noise removed estimate of the transfer function matrix then gives the following expression:

$$
\widetilde{\mathbf{H}}_{r}=\mathbf{V} \boldsymbol{\Sigma}^{-1} \mathbf{U}^{T} \mathbf{Y} \text {. }
$$

For each analysis, a threshold $\gamma$ under which the components will be removed is determined, which can help building the diagonal matrix $\Sigma_{r}^{-1}$ by introducing the following condition:

$$
\Sigma_{r}^{-1}= \begin{cases}\operatorname{diag}\left[\frac{1}{\sigma_{1}}, \frac{1}{\sigma_{2}}, \ldots, \frac{1}{\sigma_{n}}\right], & \text { if } \sigma_{n}>\gamma \\ 0, & \text { otherwise. }\end{cases}
$$

The threshold $\gamma$ takes on the role of filtering the PCs; therefore, the irrelevant ones are not taken into account in the analysis, and the engineers can focus on improving the areas that are related to the major contributors. In the following section, the advantage of the crosstalk cancellation technique over the least-square method is demonstrated with a numerical example.

2.2.3. Comparative Study of OTPA Methods. Based on above analysis, the traditional OTPA and the improved OTPA are comparatively studied, and the differences are listed below:

(1) The least-square only method does not allow for source contribution analysis because it does not use the SVD to cut the transfer matrix into major components. Since those components are used in the source contribution analysis, the user cannot know the contribution of each source without this information.

(2) The least-square only method does not address crosstalk or noises as well because all the frequency components and more particularly all the noises are included in the analysis, normally leading to inaccurate results. The differences are summarized in Figure 1.

\section{Numerical Case Study}

3.1. Introduction of Spherically Radiating Sources. The OTPA analysis is commonly conducted in the frequency domain for the sake of simplicity. That is why we define the sources and the responses at the receivers in the frequency domain as well. As shown in Figure 2, there are two sound pressure sensors and two spherically radiating acoustical sources

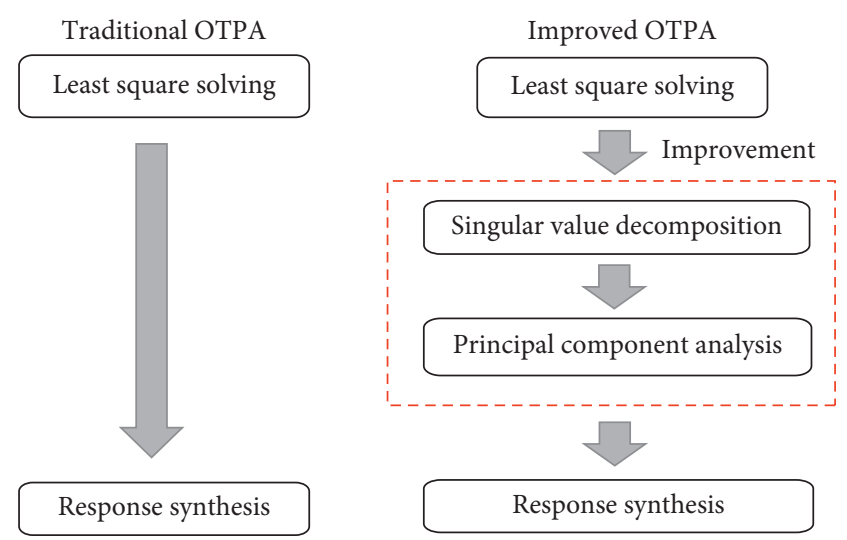

FIGURE 1: Framework of the traditional OTPA vs the improved OTPA.

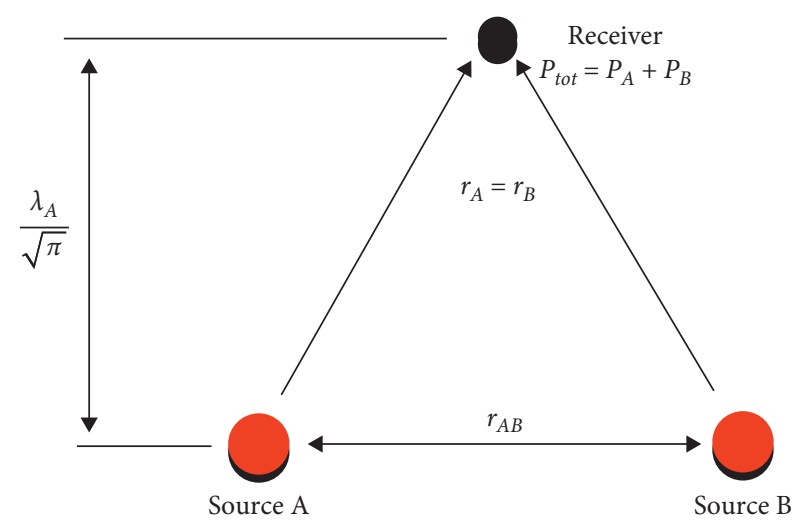

Figure 2: The general setup of two sources and the receiver.

named A and B. The sound pressure level from source A can be described as follows:

$$
\widehat{p}_{A}(r, \omega)=v_{A} \frac{j \omega \rho_{0} a^{2}}{1+j k_{A} a} \cdot \frac{e^{-j k_{A}\left(r_{A}-a\right)}}{r_{A}},
$$

where $\widehat{p}_{A}$ is the sound pressure level of the source, which is obtained by taking the common logarithm of the ratio for the effective value of the sound pressure to be measured and the reference sound pressure, and then multiplied by 20. $v_{A}$ is the complex angular frequency. $\rho_{0}$ is the density of the surrounding environment, and in this study, it is air $\left(\rho_{0}=\mathbf{1}\right)$. $a$ is the radius of the spherical source, $k_{A}$ is the wave number where $k_{A}=\omega / c=2 \pi / \lambda$, ( $\lambda$ is acoustic wavelength), $c$ is the speed of sound in the void, and $r_{A}$ is the distance from source A to its measurement point.

The total sound pressure obtained at the receiver can be seen as the addition of the two sources' contribution vector:

$$
\begin{aligned}
\widehat{p}_{\text {total }}(r, \omega)= & v_{A} \frac{j \omega \rho_{0} a^{2}}{1+j k_{A} a} \cdot \frac{e^{-j k_{A}\left(r_{A}-a\right)}}{r_{A}} \\
& +v_{B} \frac{j \omega \rho_{0} b^{2}}{1+j k_{B} b} \cdot \frac{e^{-j k_{B}\left(r_{b}-b\right)}}{r_{b}} .
\end{aligned}
$$

In the numerical case study, two different aspects of the problem have to be taken into consideration. The first one is 
the crosstalk phenomenon between the input sources, modeled by two measurement points (sensors in an experimental setup), one per source, which are both placed at a distance $\lambda_{A} / 16$ from the respective sources. The total contribution at each sensor is equal to the contribution of both sources, and the crosstalk study layout is represented in Figure 3. Secondly, the general setup is represented by the two sources distant from each other by a value $r_{A B}$. The receiver is placed at a distance $r_{A}$ from source $\mathrm{A}$ and $r_{B}$ from source B. Unless mentioned otherwise, $r_{A}=r_{B}$; therefore, the distance from the sources can be seen as an isosceles triangle and summarized as one distance $\lambda_{A} / \sqrt{\pi}$, which is shown in Figure 3.

Dimensions are only considered in one plane for simplicity, and the flowchart of the proposed OTPA is described in Figure 4.

3.2. Determining the Optimal OTPA Parameters. In this numerical study, the goal is to reproduce a virtual model similar to the available test bench. For instance, the radii of the sources $A$ and $B$ are defined at $\lambda_{A} / \mathbf{9 5}$, which corresponds to a $150 \mathrm{~mm}$ sized spherical source. It is a close approximation of the small electrical motors that are attached to the test bench presented in Section 4.

The receivers which are sound pressure sensors are placed at a distance $\lambda_{A} / \mathbf{1 6}$ and $500 \mathrm{~mm}$ away from the center of the vibrating spheres. Therefore, all the parameters are comparable to a real condition test setup and shown in Table 1.

The response receiver is equidistant from the sources to simplify computation, whose distance is automatically calculated via the angle of triangle they form, and the distance between both sources is $\lambda_{A} / \sqrt{\pi}$. Firstly, to determine the optimal threshold $\gamma$, a preliminary study is conducted, in which the influence of the number of reference measurement points per source is observed at $\gamma=1 \%, \gamma=5 \%$, $\gamma=10 \%, \gamma=15 \%, \gamma=25 \%$, and $\gamma=40 \%$ for a total of 8 measurement points per source. It is found that, respectively, all the measurement points, 7, 5, 3, 1, and none are filtered by PCA. The results are displayed in Figure 5. It is noticeable that $100 \%$ of the contributions are included with a $1 \%$ threshold. However, this means that the smaller PCs, i.e., crosstalk, are also included. Therefore, the results obtained with such a small threshold would not be of much use. From $\gamma=\mathbf{5} \%$, a difference is noted, and two major PCs that stand above the $20 \%$ contribution line appear in the analysis.

Figure 6 represents the contributions taken into consideration during the analysis according to the threshold percentage. Comparing all the results, a threshold of 5\% is chosen to conduct all the analysis. To test the proposed method, TPA is conducted on 6 different measurement blocks, and the response synthesis results are determined in Figures 7 and 8.

From Figures 7 and 8 , it can be found that no matter what the input data block is, the synthesized response matches well with the real one, and Figures 7 and 8 are also visible around $15 \mathrm{~Hz}$ and $20 \mathrm{~Hz}$, which validates the numerical study effectively. For each block, two peaks are

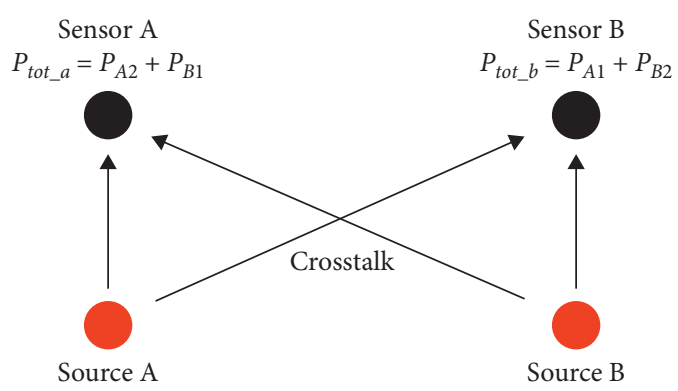

FIGURE 3: The crosstalk phenomenon represented in the numerical study.

situated at about $15 \mathrm{~Hz}$ and $20 \mathrm{~Hz}$. Therefore, the consistency of the peaks shows repeatability.

3.3. Comparisons of the Traditional and Proposed OTPA. Figure 9 is an illustration of the errors found in the traditional and proposed OTPA: the errors of the proposed method for data blocks $1,2,3,4,5$, and 6 are $1.56 \%, 1.34 \%$, $1.62 \%, 2.02 \%, 1.97 \%$, and $2.09 \%$, while the errors of the traditional OTPA method in the same data block are 5.61\%, $6.12 \%, 5.96 \%, 5.58 \%, 6.31 \%$, and $5.94 \%$, correspondingly. Comparing the results of these two methods, it is observable that the proposed OTPA is in average $4.15 \%$ more accurate than the traditional OTPA method, which is due to the noise elimination from the study as well as the crosstalk cancellation.

\section{Experimental Case Study}

In order to verify the results obtained in the numerical study, an experimental study is conducted. The measurement has been done on a cylindrical shell structure made of steel, which is designed for experiments on vibration and noise. In order to correspond to the OTPA model proposed above, two adjustable speed motors are placed on a flat plate inside the test bed as the excitation, and eight typical positions on the surface of test bed served as reference points where acceleration sensors are placed. Besides, two microphones are placed outside of the test bed, which are used as target points of OTPA. Figure 10 shows the test bed, and Figure 11 shows the positions of the sensors. Table 2 shows the equipment used. Tables 3 and 4 show the experimental parameters as well as the operating conditions.

From Figures 10 and 11, the test bed is constituted of a main cylindrical shell structure made of steel, and the sensors used are 3 single-axis accelerometers. The motors A and $\mathrm{B}$ are electrical motors. For motor A, a rotational speed of $20 \%, 60 \%$, and $100 \%$ correspond, respectively, to real $5.8 \mathrm{~Hz}, 12.4 \mathrm{~Hz}$, and $17.4 \mathrm{~Hz}$. For motor $\mathrm{B}$, a rotational speed of $50 \%$ and $100 \%$ correspond, respectively, to real $9 \mathrm{~Hz}$ and $16.8 \mathrm{~Hz}$.

The results of the contribution analysis are shown in Figure 12. To calculate the measured contribution of each source, all percentages are added and each source is weighted out according to its speed in each block and its maximum running speed. The synthesized contribution for motor A 


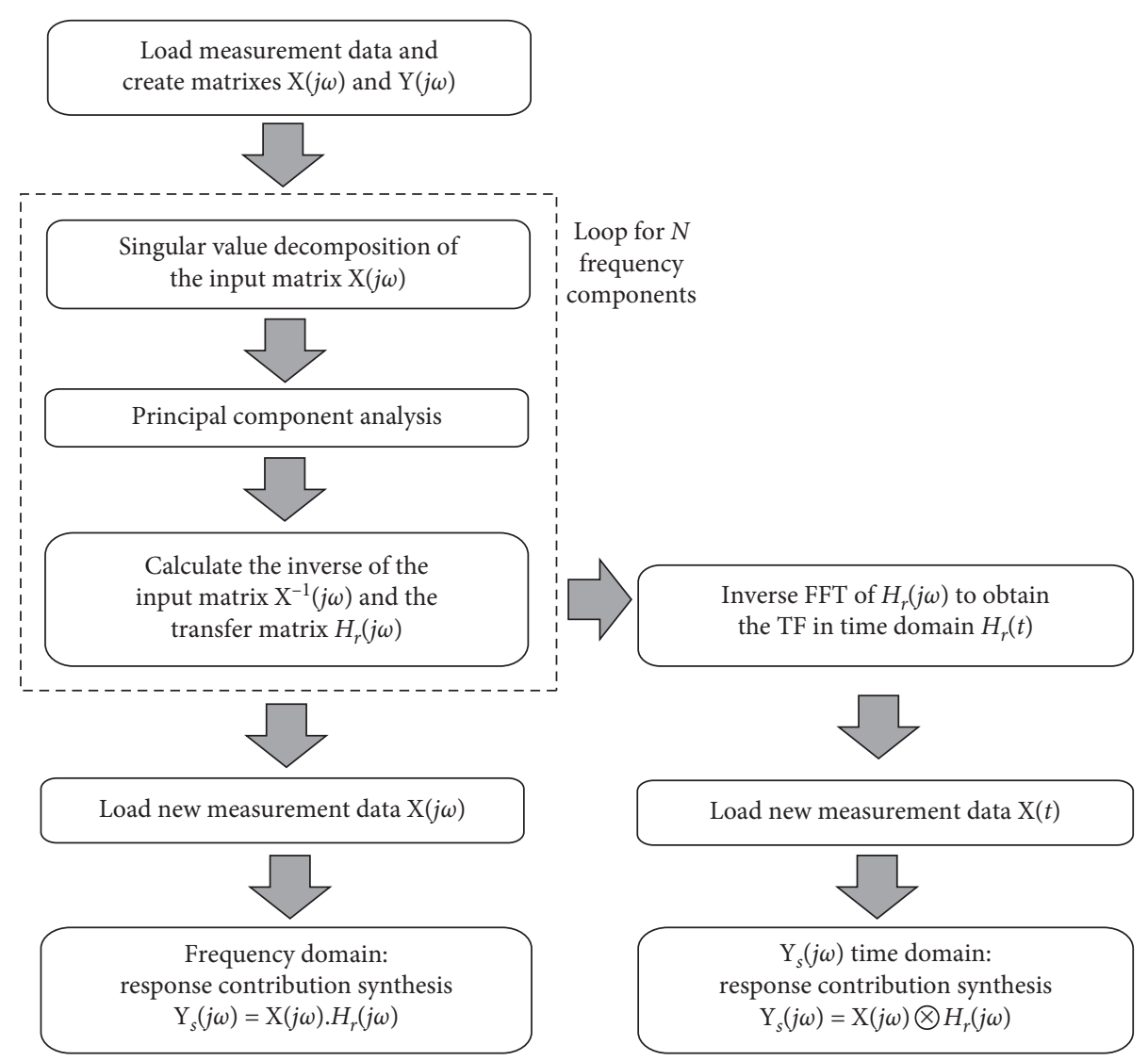

FIgUre 4: The flowchart of the proposed OTPA.

TABle 1: Parameters used in the numerical study.

\begin{tabular}{lc}
\hline Parameters & Values $(\mathrm{mm})$ \\
\hline$r_{A}=r_{B}$ & $\lambda_{A} / \sqrt{\pi}$ \\
Radius of the sources & $\lambda_{A} / 95$ \\
Distance source MP & $\lambda_{A} / 16$ \\
$\lambda_{A}$ & 1495 \\
Threshold & 5 \\
\hline
\end{tabular}

and motor B is $74.92 \%$ and $25.08 \%$. From Figure 12, a contribution of $73.20 \%$ is obtained for the motor A and $26.80 \%$ for the motor B. Therefore, it can be concluded that only absolute $1.72 \%$ errors are found between the synthesized one and the measurement. Figure 13 shows the response synthesis of the results, and the errors described in Figure 14 are obtained by comparing the measured and synthesized curves using the weighted least-square (WLS) method described as follows:

$$
\varepsilon=\frac{1}{n} \sum_{i=1}^{n}\left|\mathbf{1}-\frac{\text { synthesized value }}{\text { theoritical value }}\right| \text {. }
$$

The WLS allows users to determine point by point how both curves differ. From Figure 14, fairly small errors are obtained with only $2.13 \%$ for the first data block and $1.98 \%$ for the second data block. Furthermore, the proposed OTPA is $4.20 \%$ more accurate than the traditional OTPA method. Indeed, the proposed OTPA has an average error of $2.06 \%$, while the traditional OTPA has an average error of $6.26 \%$, which means that the proposed method performs more accurately than that of the traditional OTPA method. However, to further test the adaptability of SVD- and PCAoptimized OTPA, a comprehensive study is conducted in the following chapter.

\section{The Comprehensive Study}

The comprehensive study examines the behavior of the proposed OTPA, and the effects of different parameters are also studied. Firstly, the effect of a correlation factor between the sources and then the distance between the sources and the receiver is studied. For both parameters, the study is first simulated and then reproduced on the same test bed.

\subsection{Influence of the Correlation Factor}

5.1.1. Simulation of the Correlation Influence. The first case study consists of two structure-borne sources. Each source, respectively, $\mathrm{A}$ and $\mathrm{B}$, represents a small electrical motor, with maximum rotational speeds (frequency $F_{i}$ ) of $17.60 \mathrm{~Hz}$ and $18.13 \mathrm{~Hz}$. Both sources are considered to be the same size for calculus simplification and have, respectively, an amplitude of $V_{a}=0.02$ and $V_{b}=0.01$. Their mathematical expression is summarized as follows:

$$
f_{i}(t)=V_{i} \sin \left(2 \pi F_{i} t\right),
$$




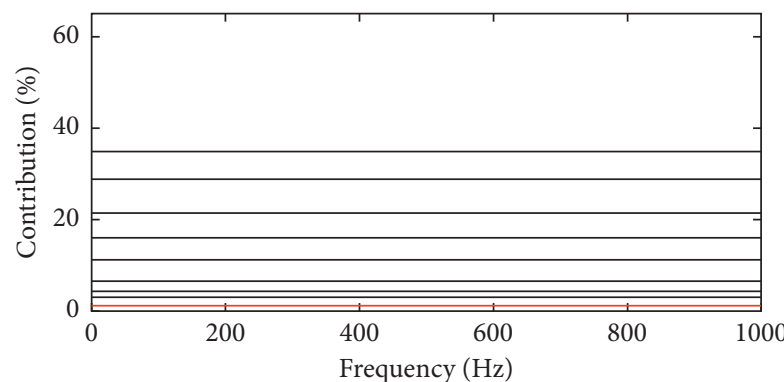

- PC

_ Threshold

(a)

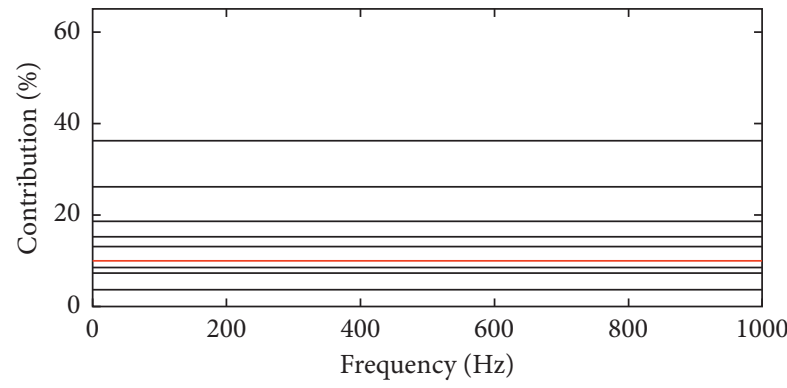

- PC

_ Threshold

(c)

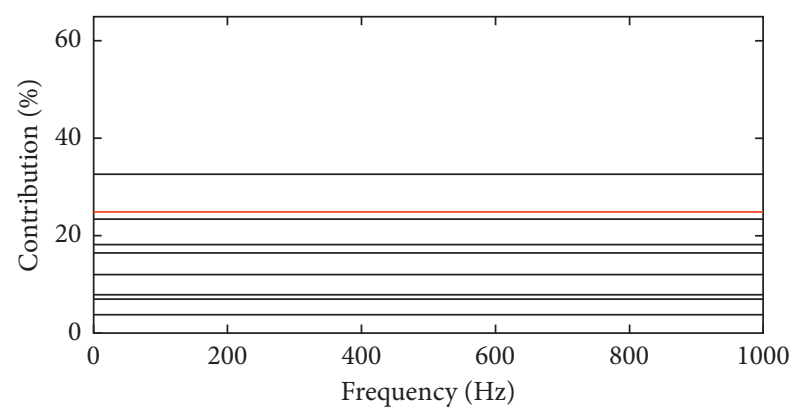

PC

Threshold

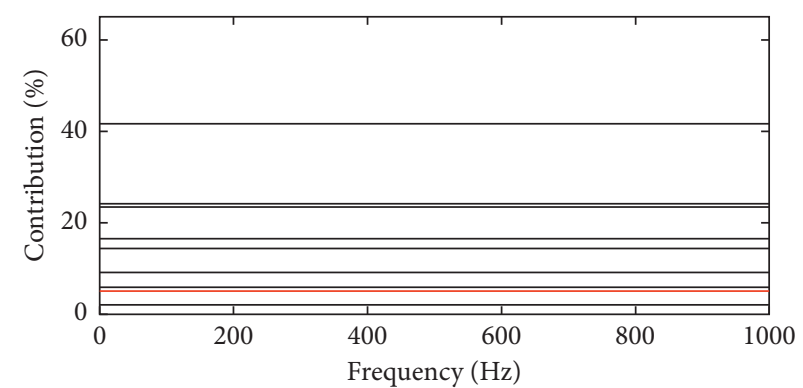

- PC

— Threshold

(b)

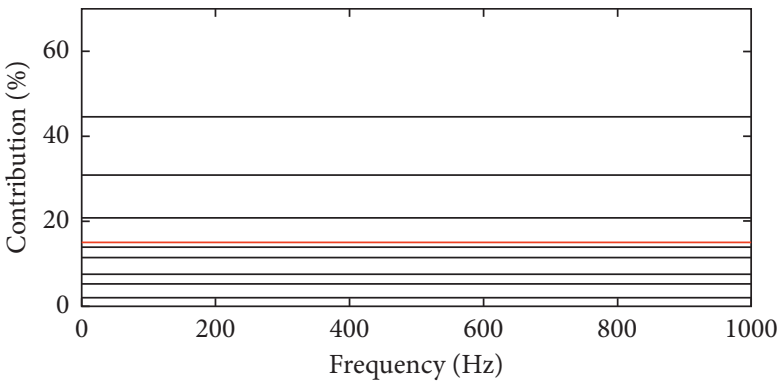

- PC

_ Threshold

(d)

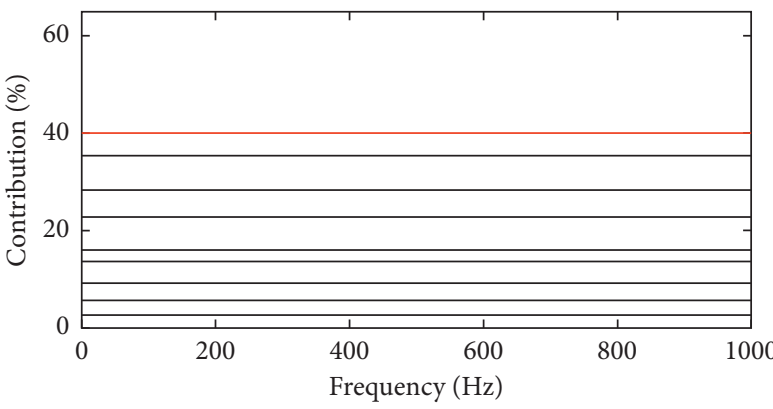

- PC

Threshold

(e)

(f)

Figure 5: Number of relevant PCs according to the threshold value. Principle component contribution: (a) $\gamma=1 \%$; (b) $\gamma=5 \%$; (c) $\gamma=10 \%$; (d) $\gamma=15 \%$; (e) $\gamma=25 \%$; (f) $\gamma=40 \%$.

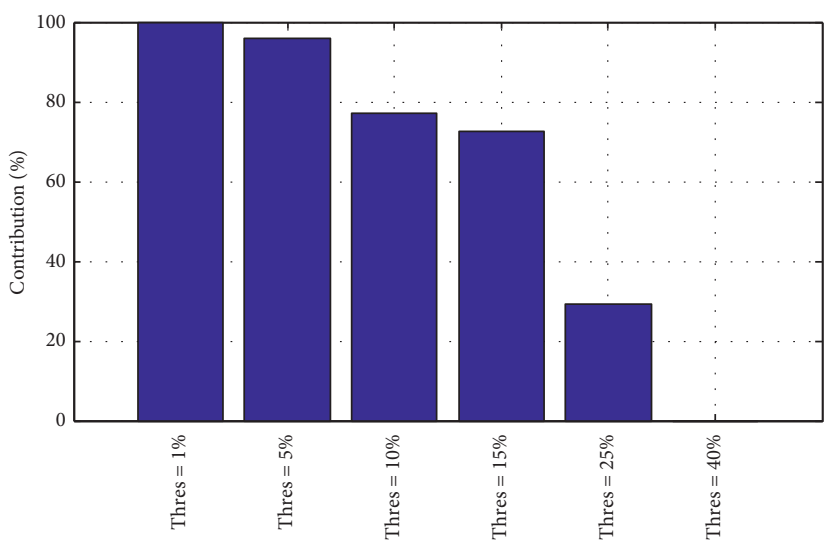

Total contribution

FIGURE 6: Total contribution of the sources according to the threshold. 


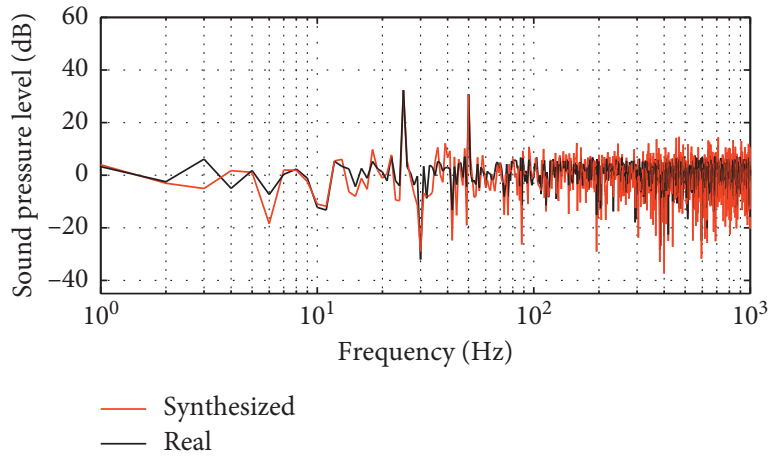

(a)

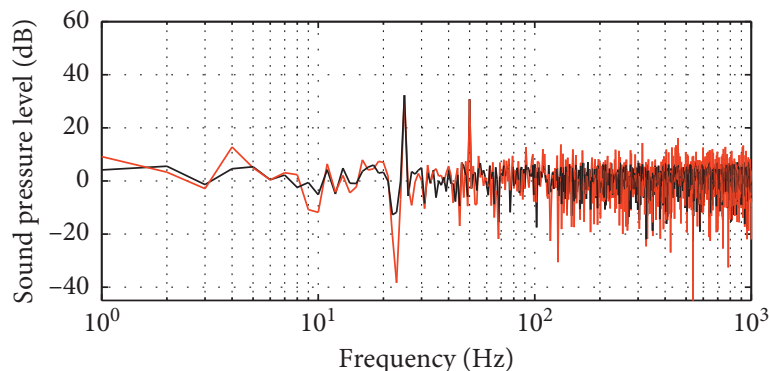

— Synthesized

_ Real

(c)

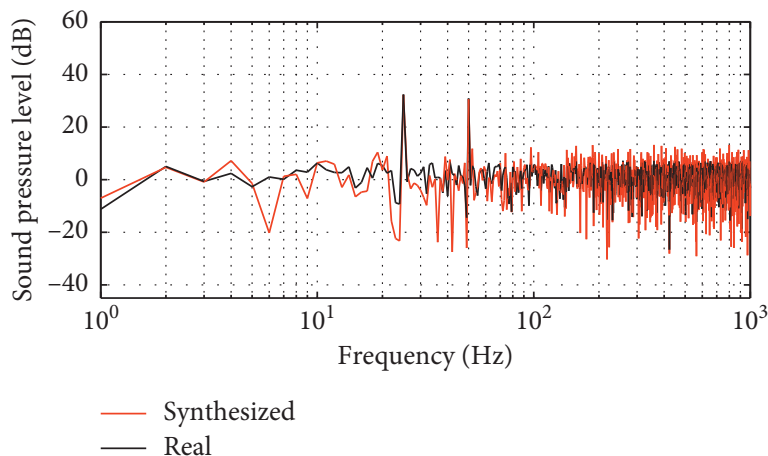

(e)

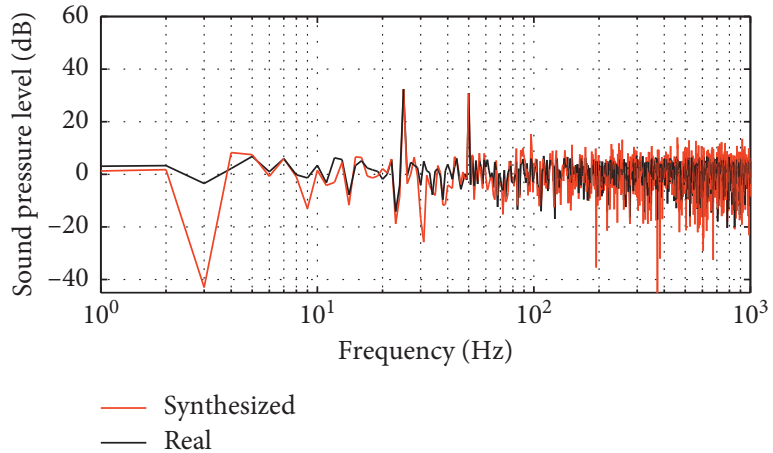

(b)

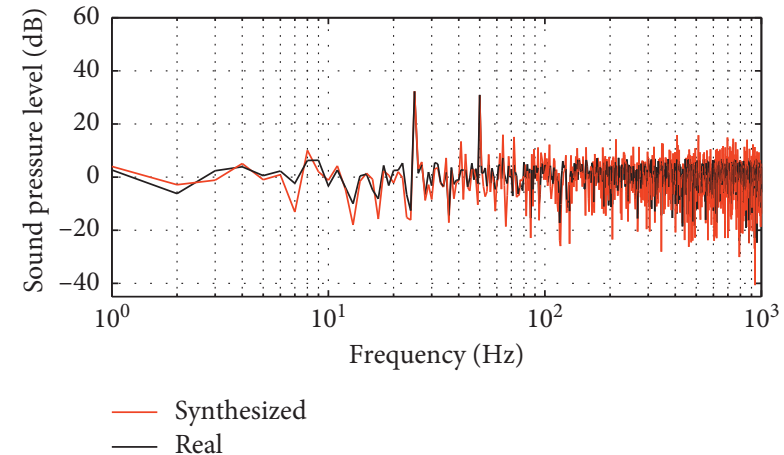

(d)

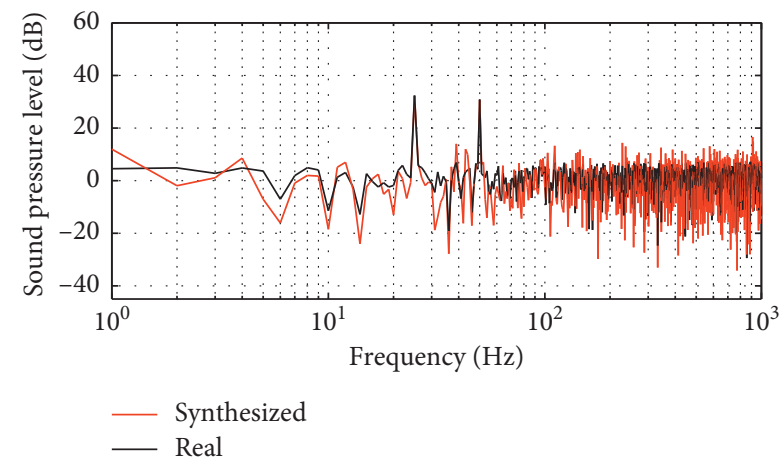

(f)

Figure 7: Sound pressure level of the numerical study for 6 different blocks: (a) measurement block 1; (b) measurement block 2; (c) measurement block 3; (d) measurement block 4; (e) measurement block 5; (f) measurement block 6.

where $f_{i}$ is the simulated signal of the source, $V_{i}$ is the amplitude of the signal, and $F_{i}$ is the rotational speed of the motor in Hertz. The signal is simulated in the time domain to easily create vibrations. Furthermore, a second harmonic is added with small amplitude to simulate the influence of the second motor on the measurement. The previous equation case then becomes as

$$
f_{A}(t)=V_{A} \sin \left(2 \pi F_{A} t\right)+V_{B} \sin \left(2 \pi F_{B} t\right) .
$$

In order to make those signals as close to the motors used in the experiment as possible, the simulated signals are compared to the measured signals of the motors. Firstly, the amplitude and frequency are determined, and then, noise is added to mimic experimental conditions. This process is described in Figure 15. Once satisfying values are found, the simulated environment is tested for different correlation factors. The correlation factor represents how similar the two motor signals are according to their frequency; for example, a correlation of $100 \%$ represents two motors running at the same frequency. It should be noted that a correlation of $0 \%$ cannot be obtained in real life; therefore, the correlation factors chosen are $20 \%, 40 \%, 60 \%, 80 \%$, and $100 \%$.

Figure 16 shows the contribution percentage of each source in function of the correlation factor. The contribution errors for correlation factor $20 \%, 40 \%, 60 \%, 80 \%$, and $100 \%$ are $1.42 \%, 5.51 \%, 6.37 \%, 16.13 \%$, and $22.62 \%$, which means the overestimation of source $\mathrm{A}$ and an underestimation of source $\mathrm{B}$ as the correlation factor increases.

Due to the similarity of the two sources, the algorithm considers that they are so similar, as they are a part of the 


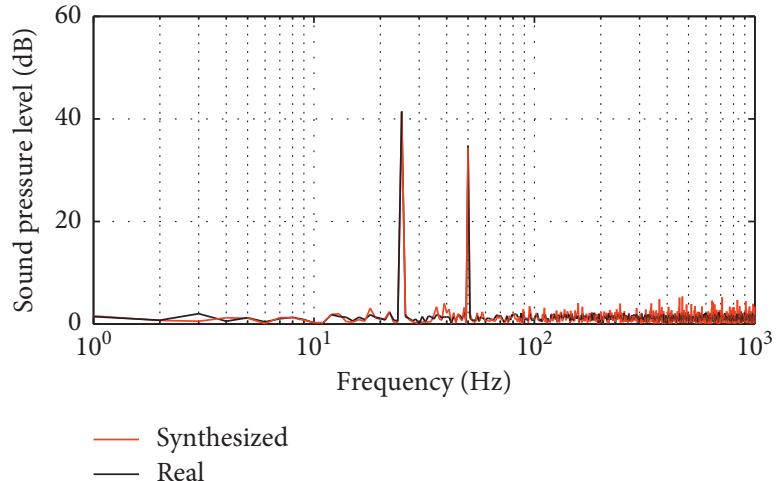

(a)

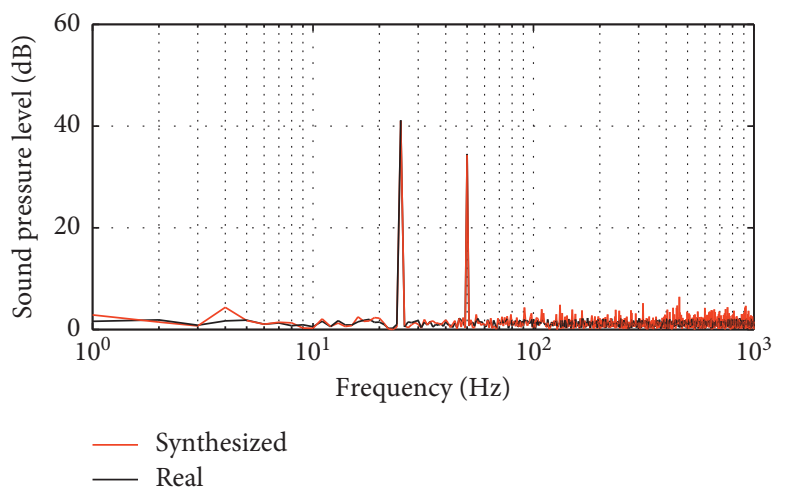

(c)

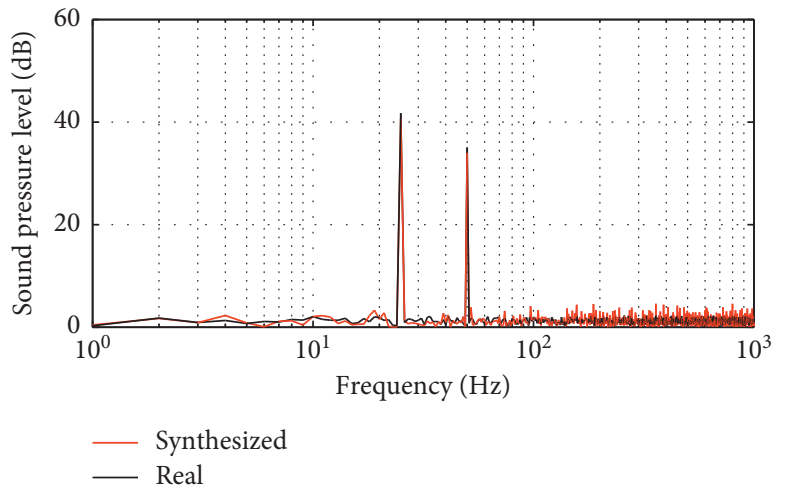

(e)

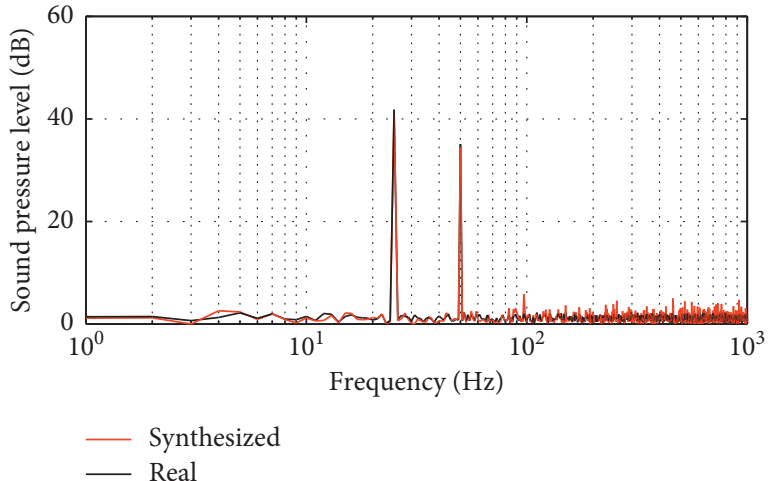

(b)

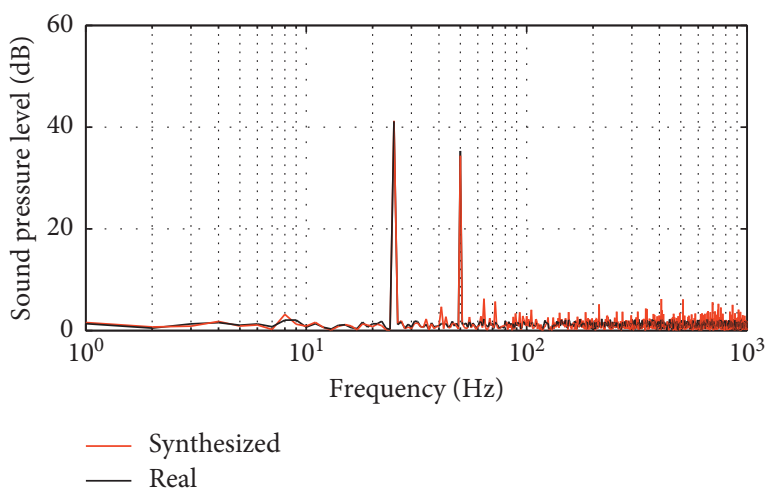

(d)

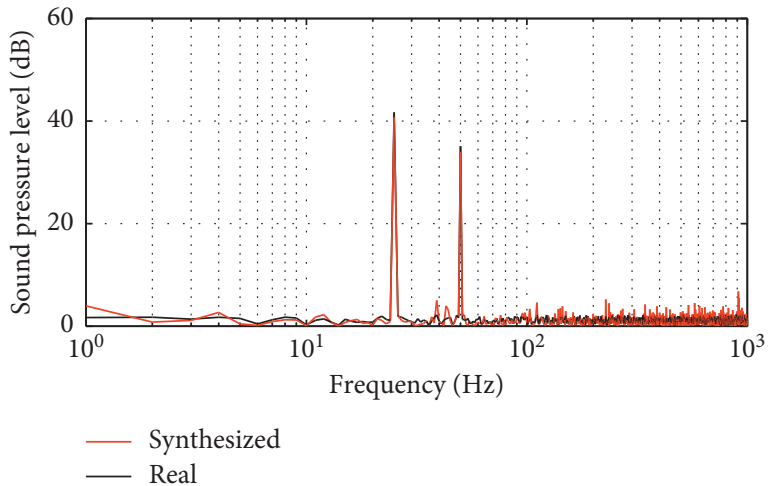

(f)

Figure 8: Spectrum of the numerical study for the 6 corresponding blocks: (a) measurement block 1; (b) measurement block 2; (c) measurement block 3; (d) measurement block 4; (e) measurement block 5; (f) measurement block 6.

same source, leading to the overestimation of source A and treating source B as a mathematical residue. For a smallest correlation factor, the error of $1.42 \%$ is obtained. However, when sources are similar, a staggering error of $22.62 \%$ is observable. Therefore, the more different the setup conditions are when doing an OTPA analysis, the better the transfer function can be determined, and the better the response can be synthesized. The response synthesis is shown in Figure 17. The total response synthesis is generally well transcribed no matter what the correlation between the motors is. Figure 18 shows the errors of the proposed OTPA with the traditional OTPA. For a 20\% correlation, the two curves match quite well with only a $1.32 \%$ average error for the proposed OTPA while a $9.98 \%$ average error for traditional OTPA.

Furthermore, the percentage rises slightly as the correlation increases to $100 \%$, but the error does not get higher than a mere $5.31 \%$ for the proposed OTPA, while up to $12.61 \%$ for the traditional OTPA. Generally, the proposed OTPA has a smaller average error of $3.34 \%$ than $11.22 \%$ of the traditional OTPA, which further improves the accuracy of OTPA. However, as earlier stated, the individual contribution of the sources is not accurate anymore as the correlation increases. In order to further observe this behavior and confirm the results found in the simulation, the same factors are studied in an experimental environment. 


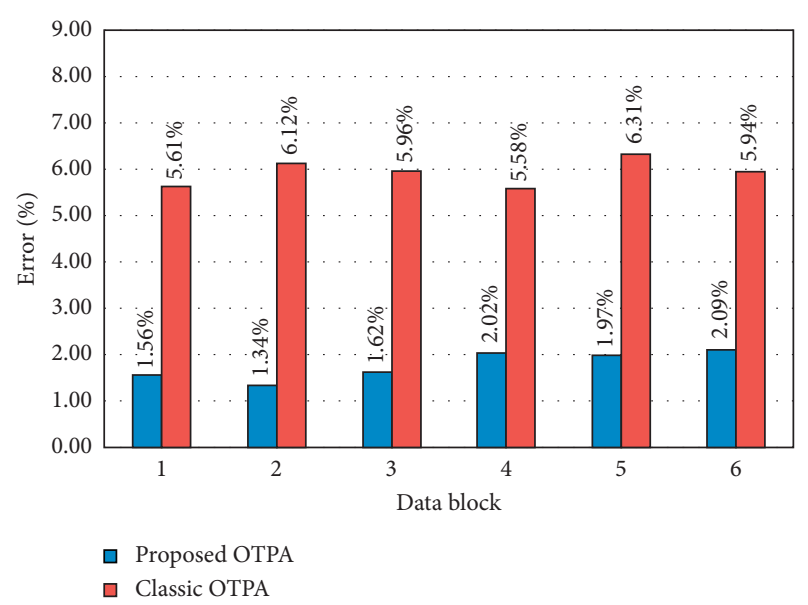

FIgURE 9: Errors of the proposed and classic OTPA in the response synthesis.

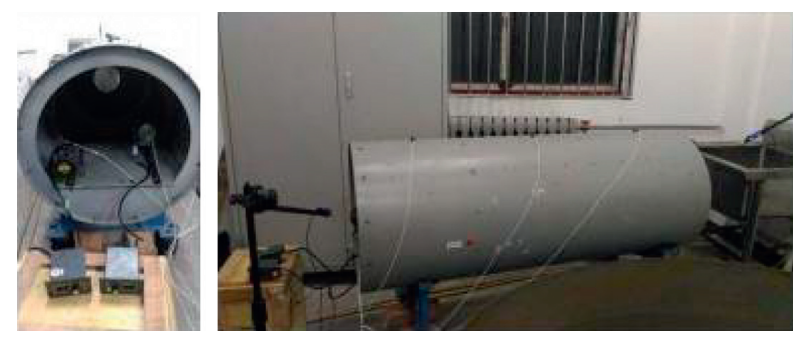

FIgURE 10: The test bench used for the experimental studies.

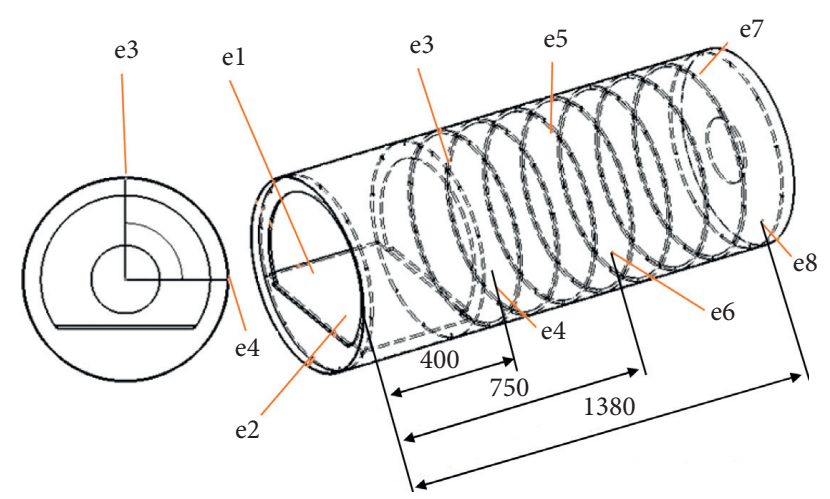

FIgURe 11: Positions of sensors used for the experimental studies.

TABLE 2: Equipment used in the experiment.

\begin{tabular}{lcc}
\hline Equipment & Quantity & Model \\
\hline Microphones & 2 & $\begin{array}{c}\text { Bruel and Kjaer prepolarized free- } \\
\text { field 1/2" microphone-type 4189 } \\
\text { BSWA TECH MPA 201 }\end{array}$ \\
$\begin{array}{l}\text { Data acquisition } \\
\text { system }\end{array}$ & 1 & Coco 80 THP systems \\
Motor & 2 & \\
\hline
\end{tabular}

5.1.2. Experimental Observation of the Correlation Factor Influence. In order to verify the simulation established in the previous section, a similar setup is tested under real life conditions. Both motor A and motor B are equipped with
TABLE 3: Experimental parameters.

\begin{tabular}{lc}
\hline Parameters & Values and units \\
\hline Sampling frequency & $1000 \mathrm{~Hz}$ \\
Data length & $10 \mathrm{~s}$ \\
Block size & 10000 \\
\hline
\end{tabular}

TABle 4: Operating conditions.

\begin{tabular}{lcccccc}
\hline $\begin{array}{l}\text { Simulation conditions } \\
(\%)\end{array}$ & $\begin{array}{c}1 \\
(\%)\end{array}$ & $\begin{array}{c}2 \\
(\%)\end{array}$ & $\begin{array}{c}3 \\
(\%)\end{array}$ & $\begin{array}{c}4 \\
(\%)\end{array}$ & $\begin{array}{c}5 \\
(\%)\end{array}$ & $\begin{array}{c}6 \\
(\%)\end{array}$ \\
\hline $\begin{array}{l}\text { Rotational speed of } \\
\begin{array}{l}\text { motor A } \\
\text { Rotational speed of } \\
\text { motor B }\end{array}\end{array}$ & 20 & 20 & 60 & 100 & 100 & 60 \\
\hline
\end{tabular}

accelerometers placed at a distance $d=100 \mathrm{~mm}$ from their respective centers. A run up test is done prior to any other measurement to obtain the true speeds of the motors. One measurement point (an accelerometer) is assigned to each source. One accelerometer representing the response is placed on top of the test bench at $750 \mathrm{~mm}$ from the front extremity of the cylinder. The general setup is shown in Figure 19. The motors are tested with a correlation factor of $20 \%$ and $80 \%$, as the experimental conditions where correlation factors that are too similar cannot be clearly observed. Figure 20 shows the experimental contribution percentage: the errors of the contribution evaluation are $25.47 \%$ and $39.21 \%$ for correlation $20 \%$ and $80 \%$, respectively, which means that there is an overestimation of the first path (i.e., source A) and an underestimation of the second path when the correlation increases, and the high error can be explained by a significant crosstalk at the sources.

The response synthesis is shown in Figure 21, and the results are similar to those of the numerical model. Figure 22 shows the errors for both methods: the synthesized responses in vibrations match quite well the measured response with an error of $3.28 \%$ for the lowest correlation $20 \%$, and $6.21 \%$ for the highest correlation $80 \%$, while the errors of the traditional OTPA are $7.03 \%$ and $11.67 \%$. Generally, the proposed OTPA has an error in average that is $4.60 \%$ lower than the traditional OTPA. Furthermore, all the results emphasize the requirement for correlation between the sources to be as low as possible when conducting an OTPA analysis. Although the correlation factor does not have much impact on the computation of the transfer matrix $H$, however, it does influence the response synthesis obviously.

\subsection{Influence of the Distance between the Receiver and the Sources}

5.2.1. Simulated Environment. This study is simulated using the same principles as in Section 3. The simulated setup is constituted of two vibrating sources radiating at a frequency similar to that of the real motors in Section 4. The effect of the distance on airborne only simulation is done on three 


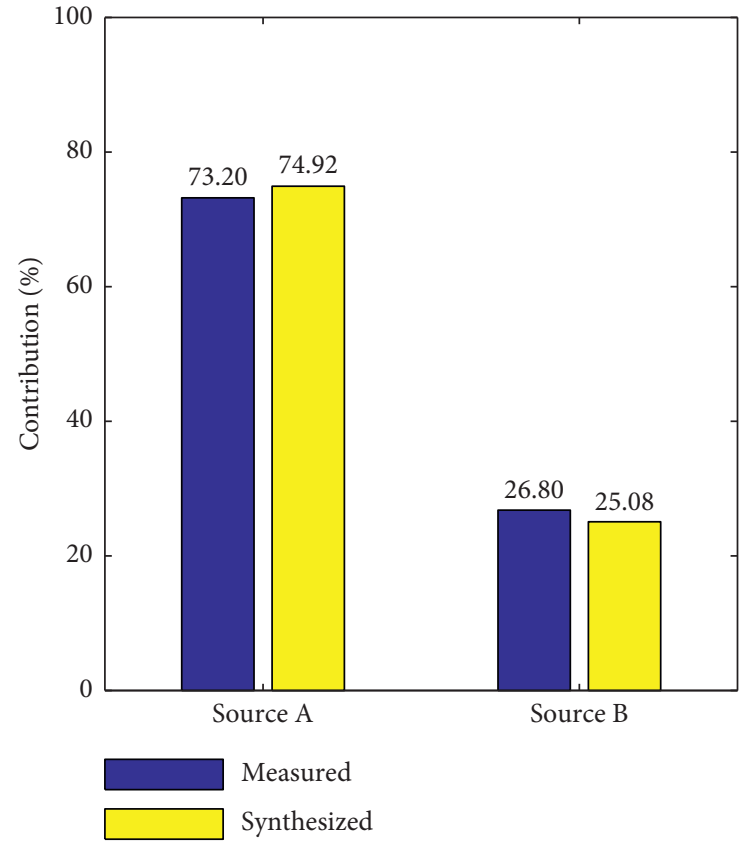

(a)

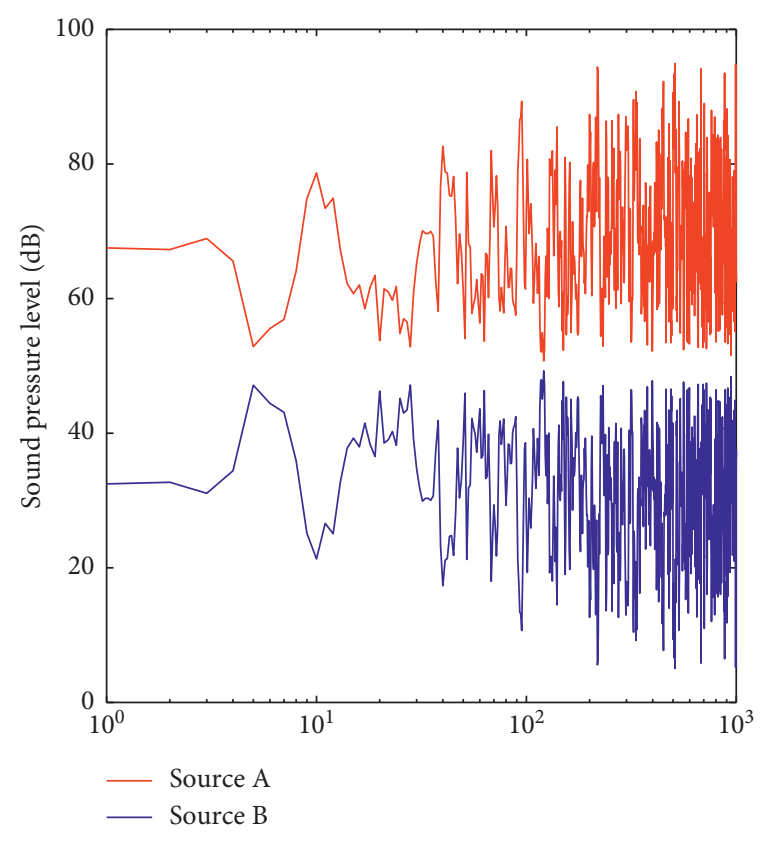

(b)

FIGURE 12: Contribution percentages of both sources with the proposed OTPA: (a) overall contribution; (b) point by point contribution.

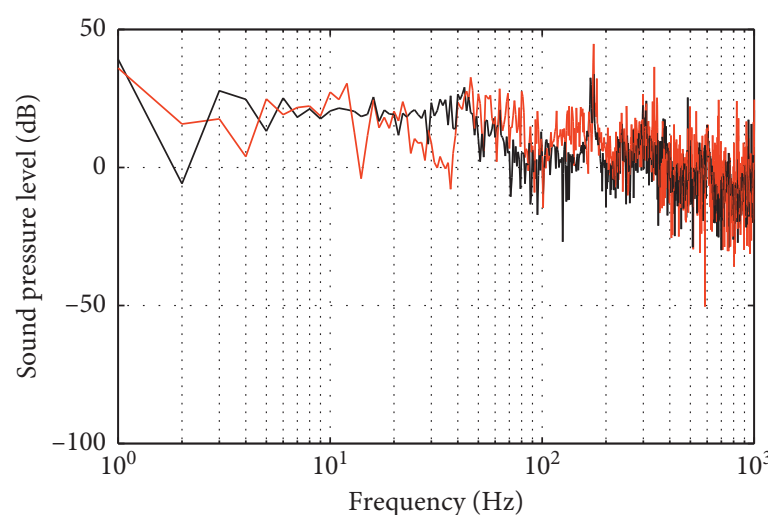

— Measured

— Synthesized

(a)

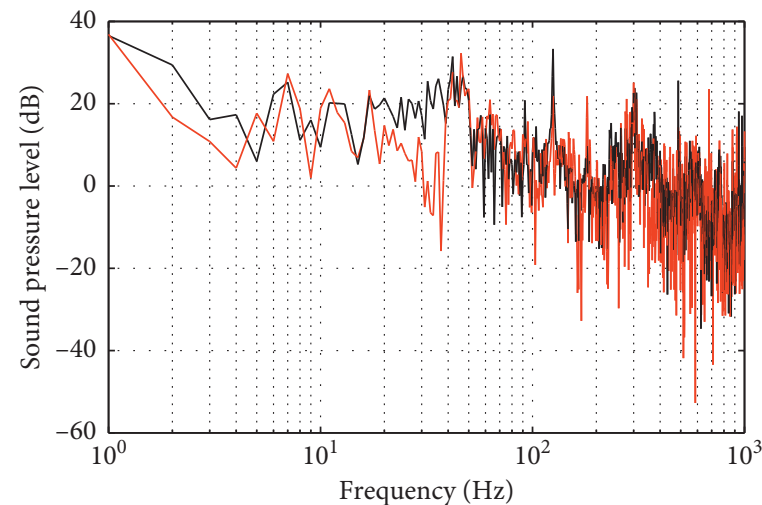

- Measured

— Synthesized

(c)

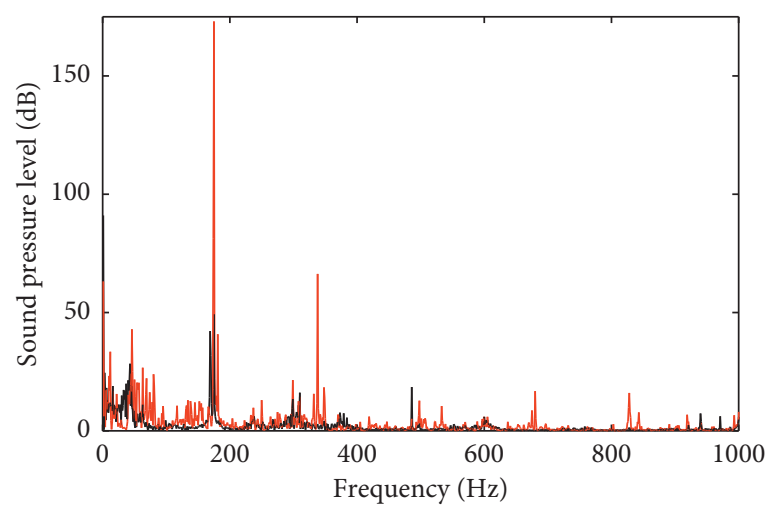

- Measured

Synthesized

(b)

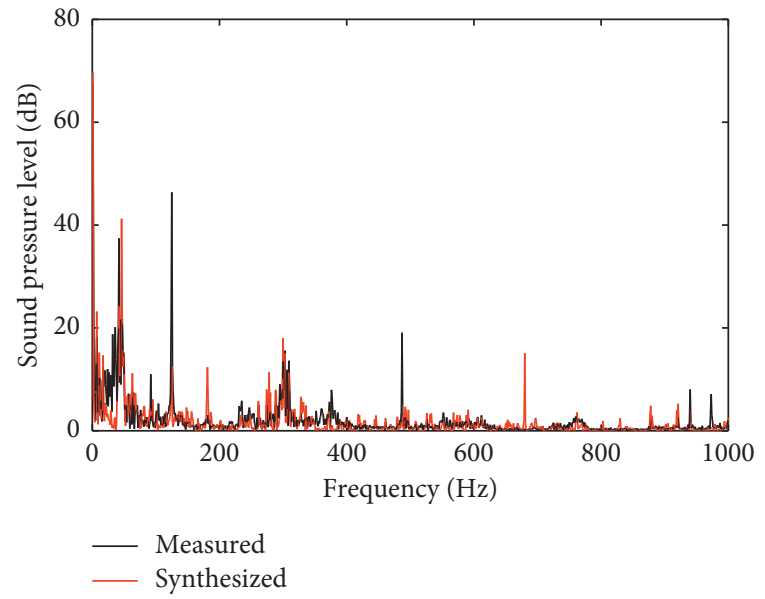

(d)

Figure 13: Response synthesis. Sound pressure level (left) and spectrums (right): (a) test measurement 1; (b) test measurement 1; (c) test measurement 2; (d) test measurement 2. 


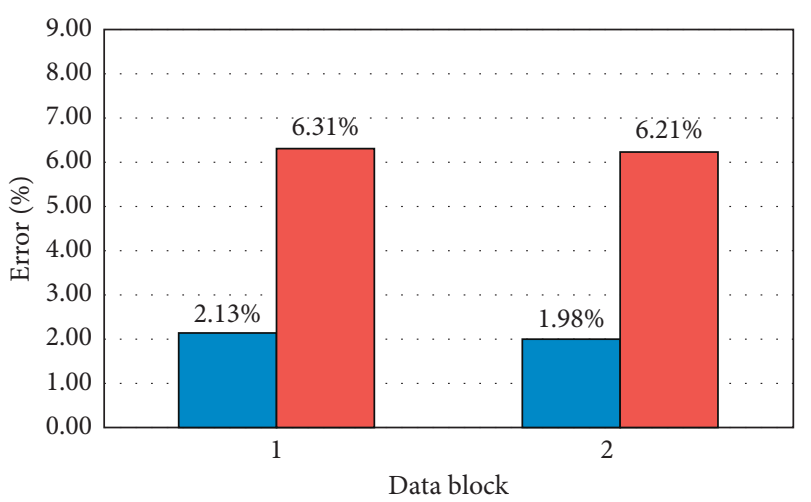

$\square$ Proposed OTPA

- Classic OTPA

FIGURE 14: Errors of the proposed and classic OTPA in the response synthesis.
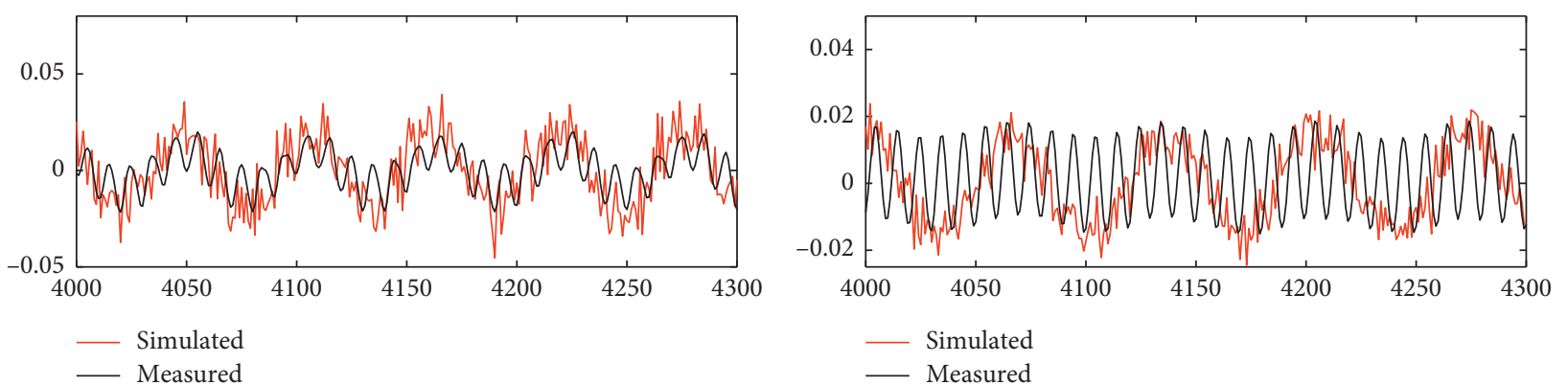

(a)

(b)

Figure 15: Measured signal and simulated source signals with noise: (a) motor A simulated vs measured at a speed of 100\%; (b) motor B simulated vs measured at a speed of $100 \%$.

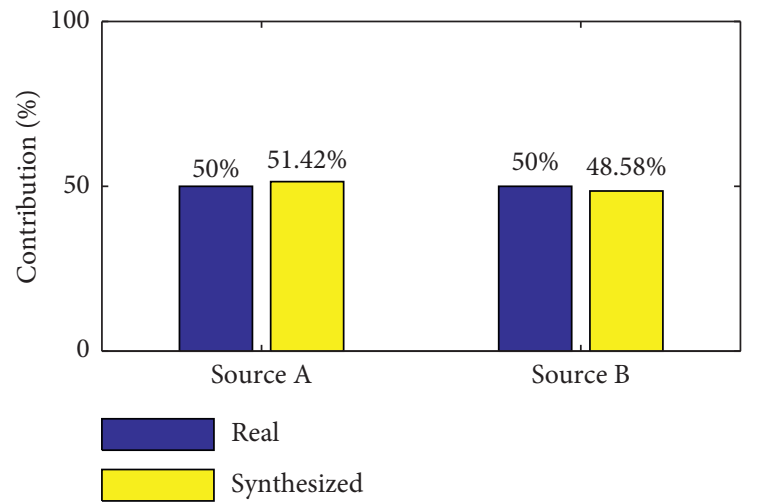

(a)

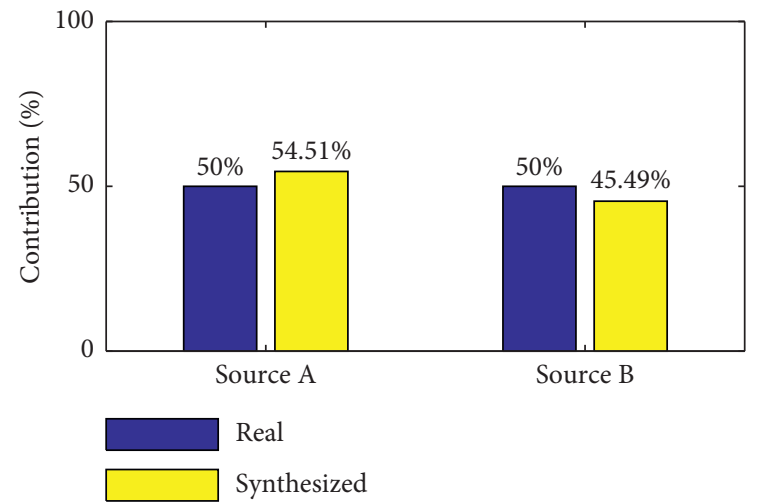

(b)

Figure 16: Continued. 


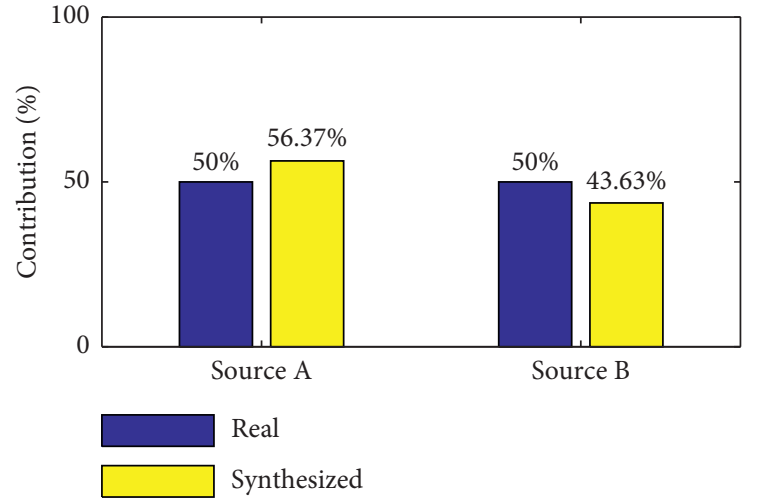

(c)

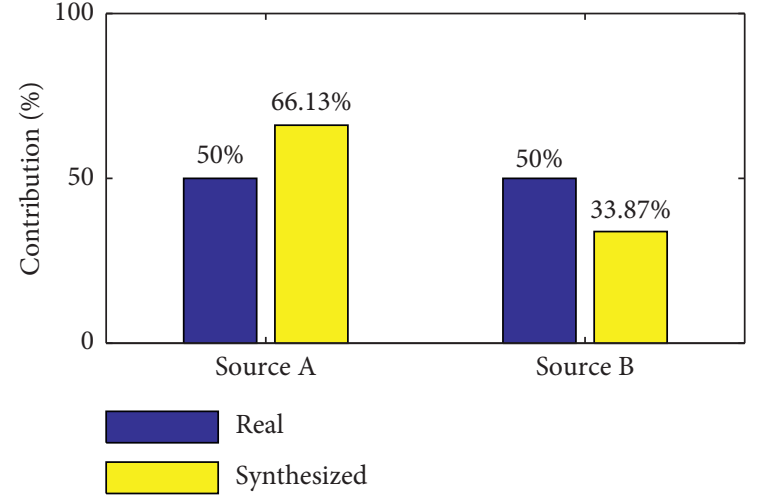

(d)

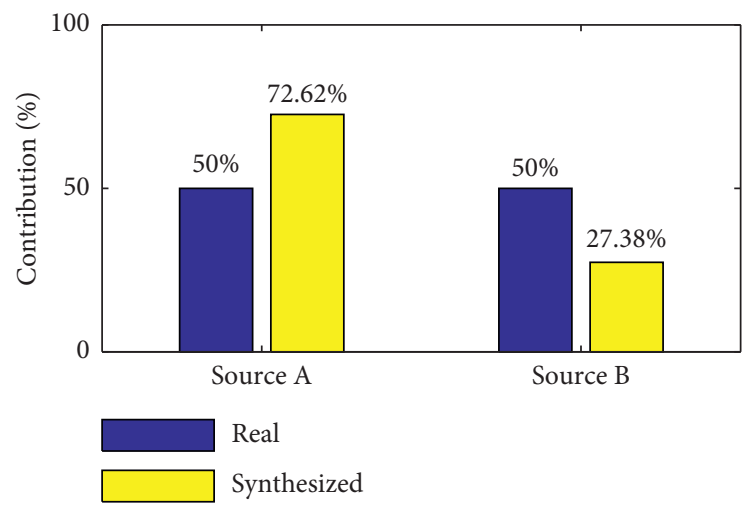

(e)

FIGURE 16: Contribution percentage of each source in function of the correlation factor: (a) correlation $=20 \%$; (b) correlation $=40 \%$; (c) correlation $=60 \%$; (d) correlation $=80 \%$; (e) correlation $=100 \%$.

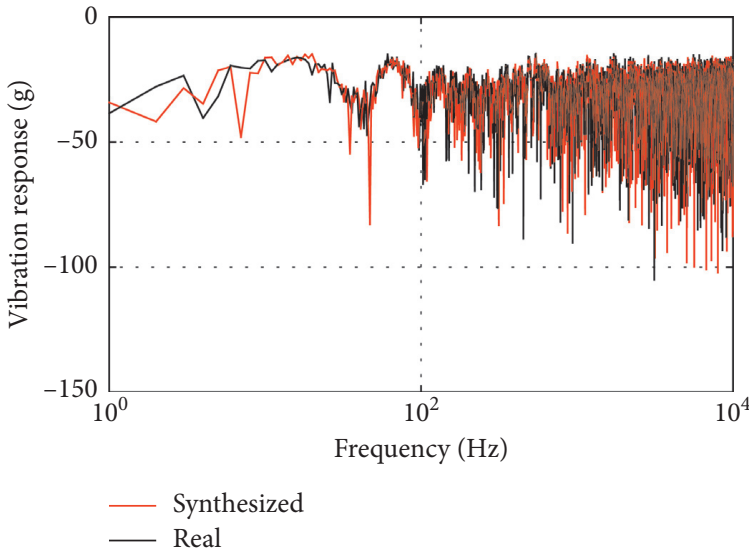

(a)

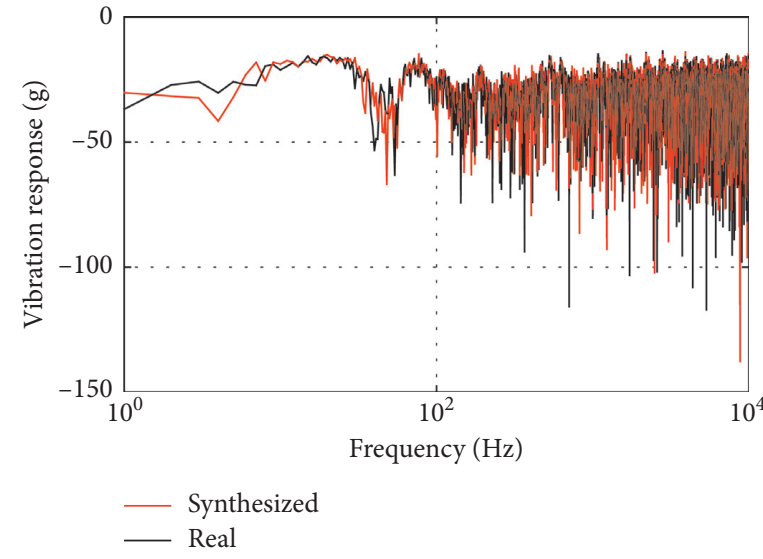

(b)

FIgURE 17: Continued. 


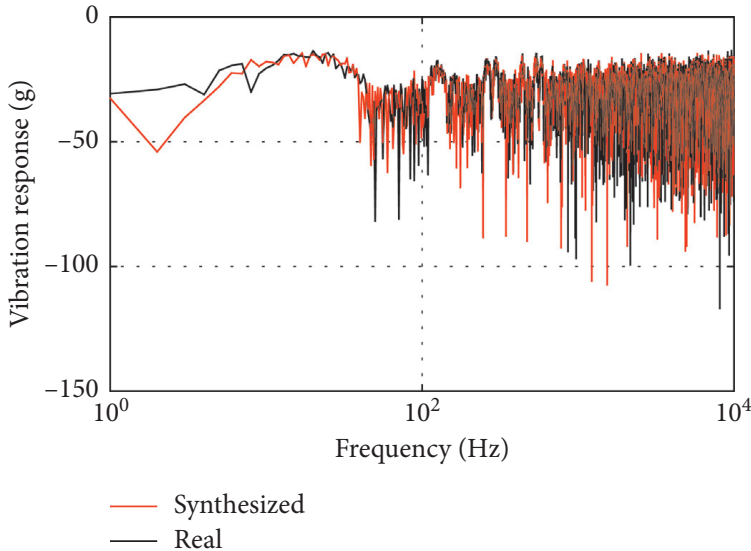

(c)

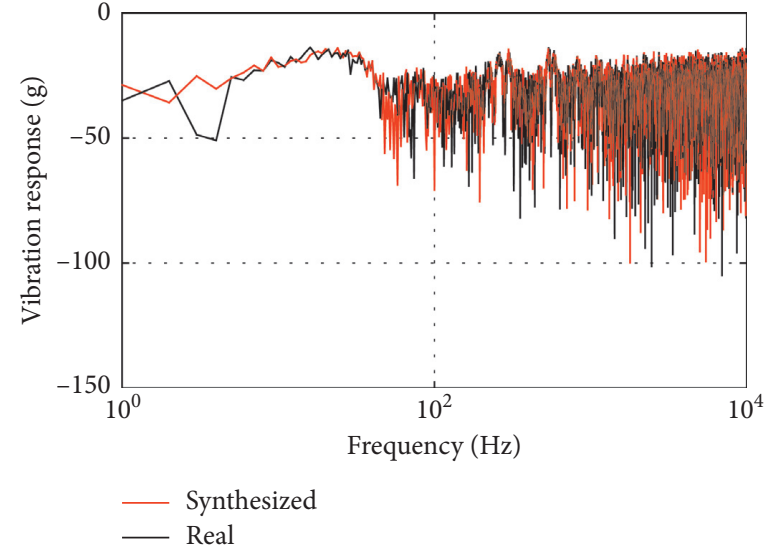

(d)

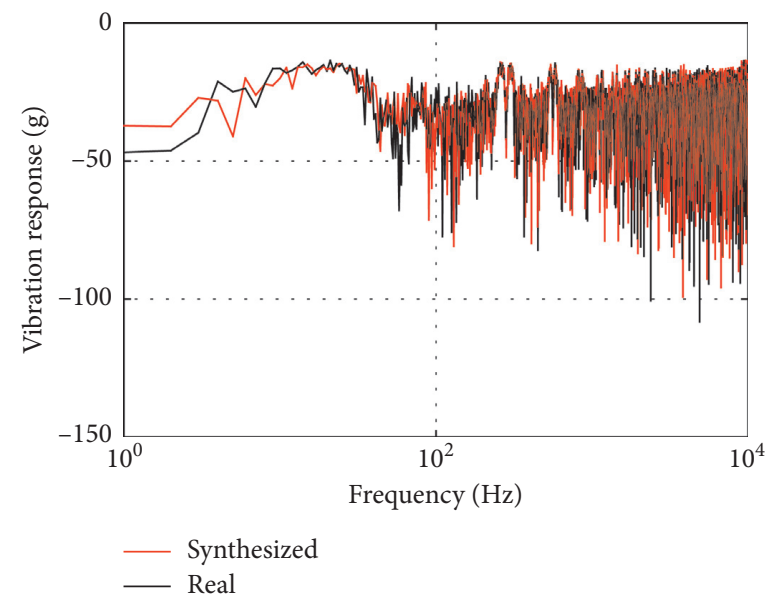

(e)

Figure 17: Total response synthesis in function of the correlation factor: (a) correlation $=20 \%$; (b) correlation $=40 \%$; (c) correlation $=60 \%$; (d) correlation $=80 \%$; (e) correlation $=100 \%$.

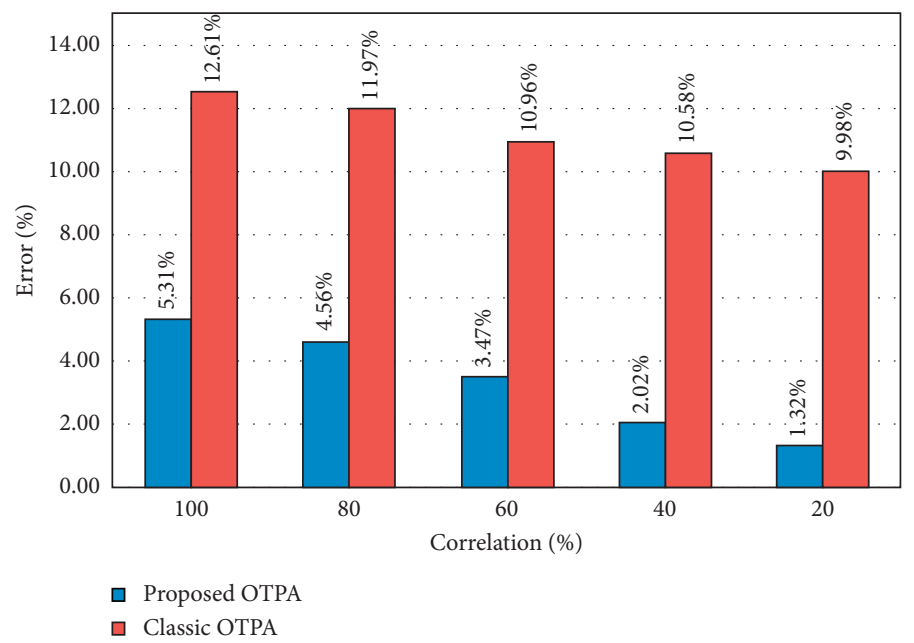

FIGURE 18: Errors of the proposed and classic OTPA in the response synthesis of the correlation study.

different distances: $d=240 \mathrm{~mm}, \quad d=750 \mathrm{~mm}, \quad$ and $d=1240 \mathrm{~mm}$ corresponding to the locations used in the experimental study to observe the same parameters.
Figure 23 details the position of the different receivers and the sources in the simulated environment, and the results are shown in Figure 24: the contribution of source A 


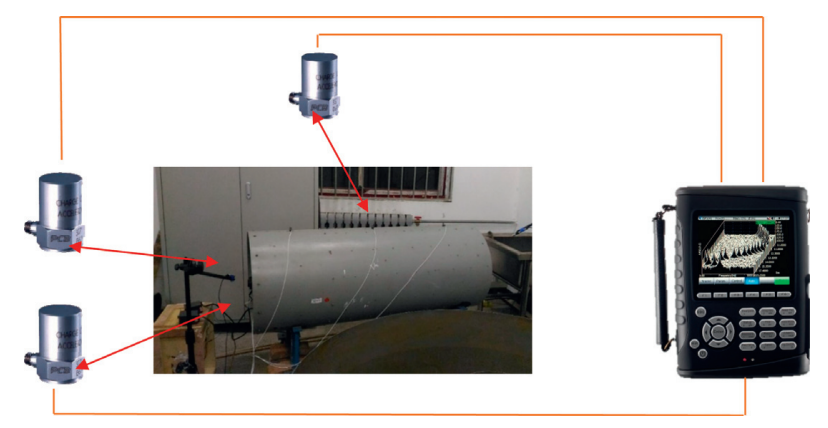

Figure 19: The setup of the experimental study.
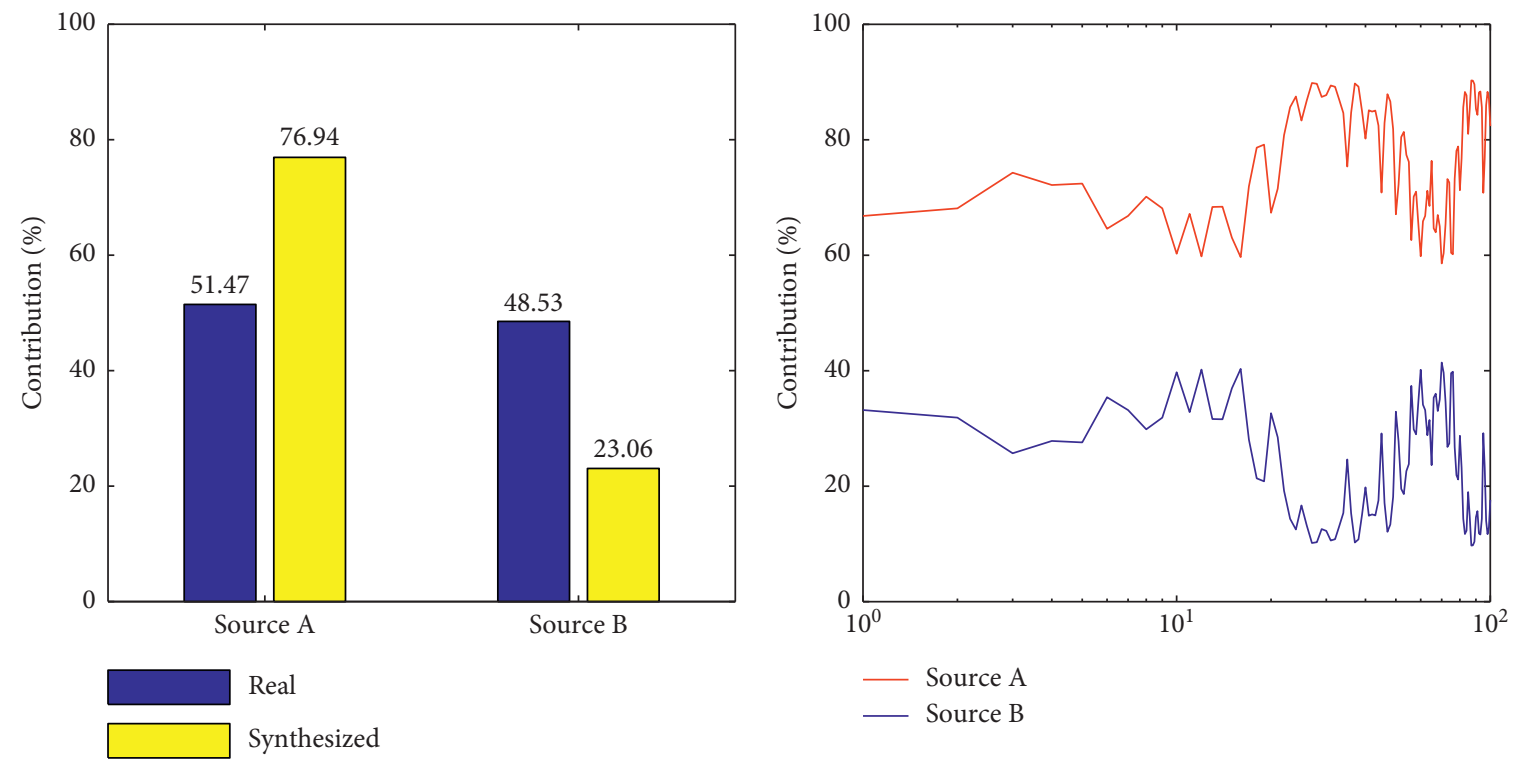

(a)

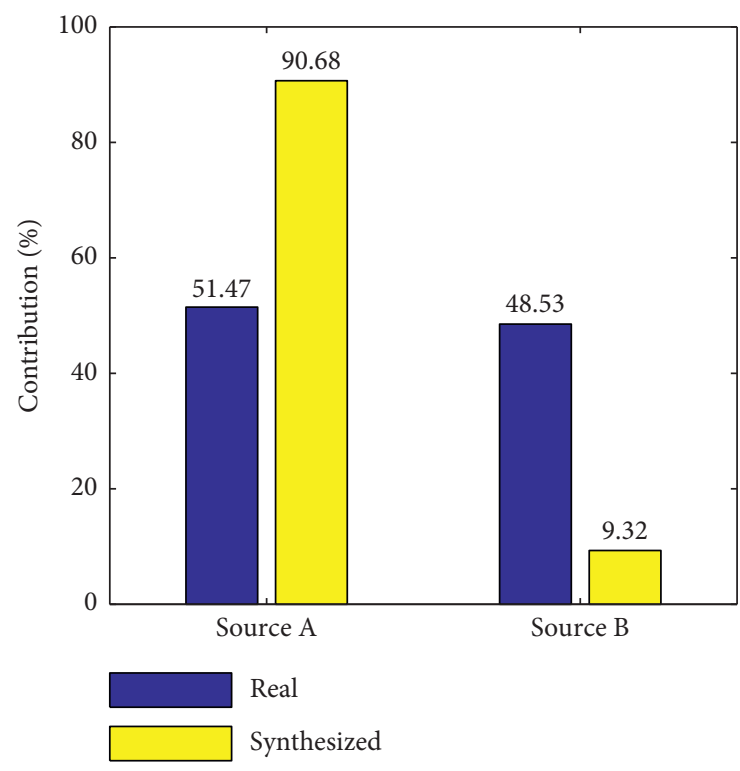

(c) (b)

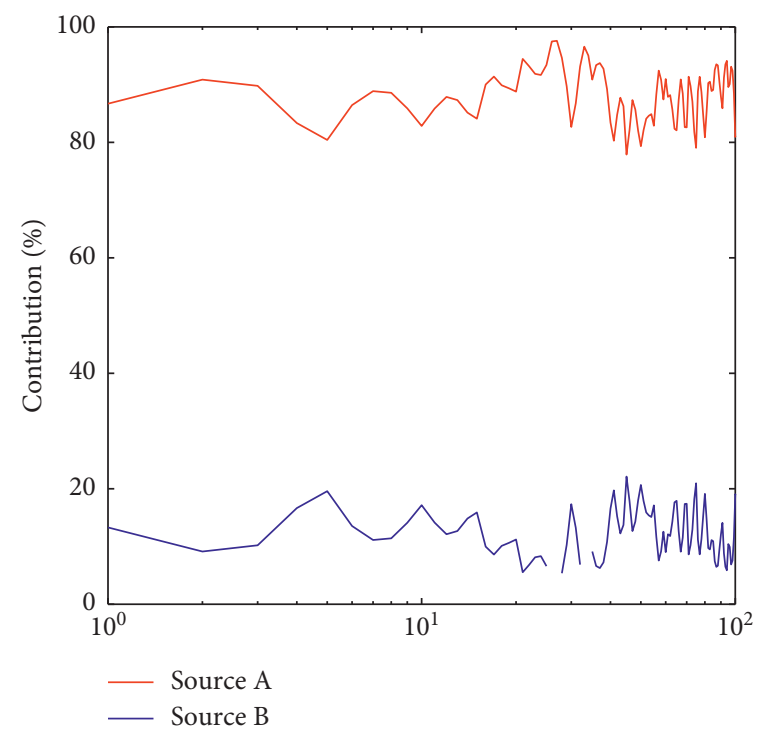

(d)

FIGURE 20: Experimental contribution percentages in function of the correlation factor: (a) correlation $=20 \%$; (b) correlation $=40 \%$; (c) correlation $=60 \%$; (d) correlation $=80 \%$; (e) correlation $=100 \%$. 


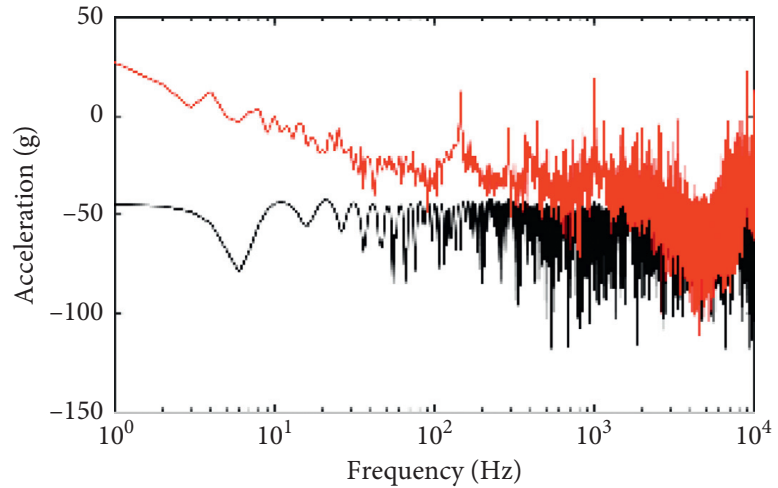

— Measured

— Synthesized

(a)

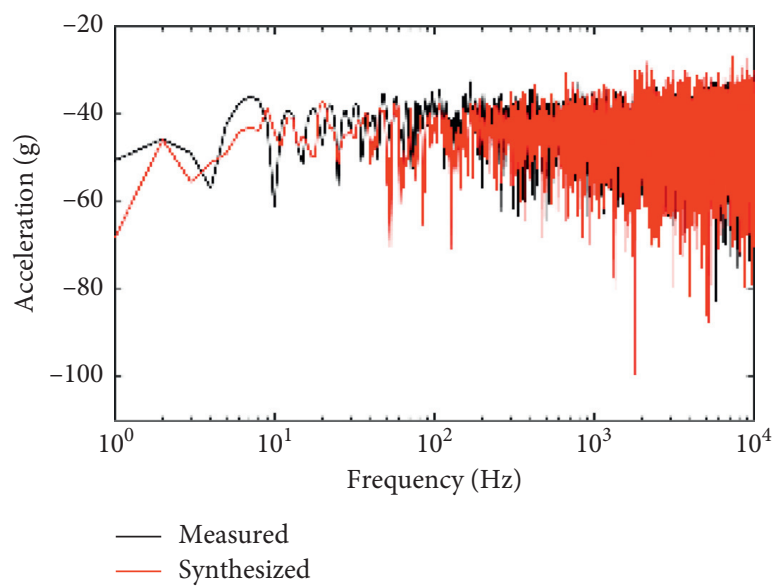

(c)

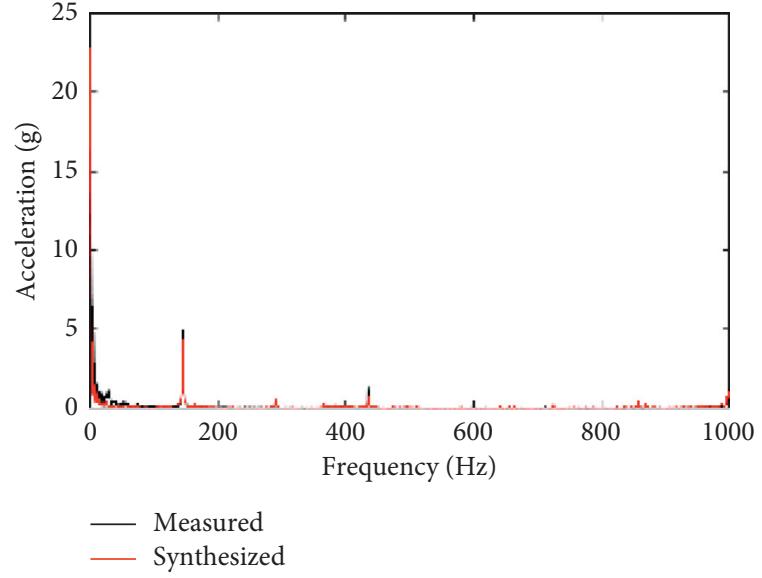

(b)

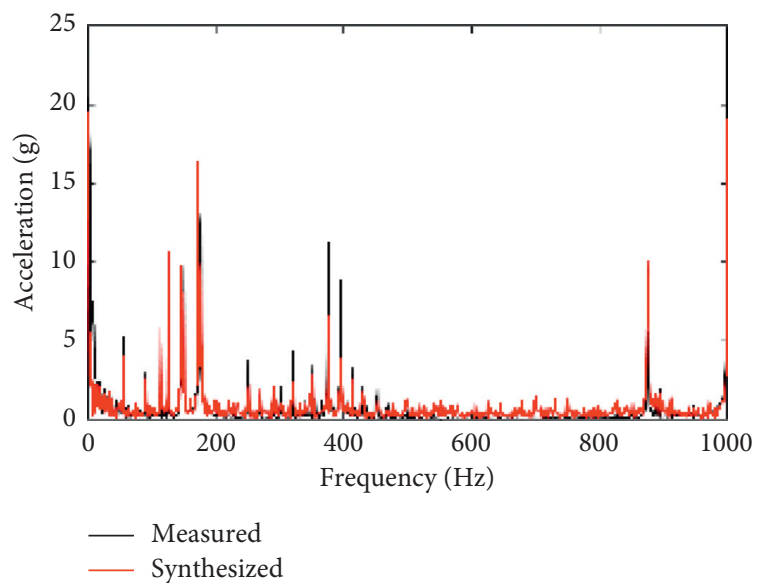

(d)

FiguRe 21: Vibration response (left) and frequency spectrum (right) at different motor correlations: (a) correlation $=80 \%$; (b) correlation $=80 \%$; (c) correlation $=20 \%$; (d) correlation $=20 \%$.

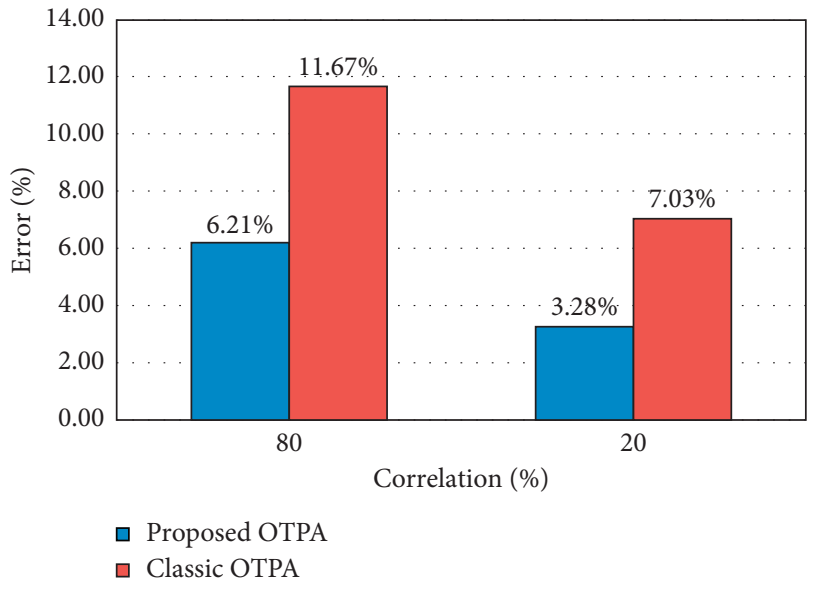

FIGURE 22: Error of the traditional and proposed OTPA according to correlation.

is $63.00 \%, 64.45 \%, 64.45 \%$, and $64.45 \%$, while the contribution of source B is $37.00 \%, 36.55 \%, 36.55 \%$, and $36.55 \%$ for theoretical, $d=240 \mathrm{~mm}, d=750 \mathrm{~mm}$, and $d=1240 \mathrm{~mm}$

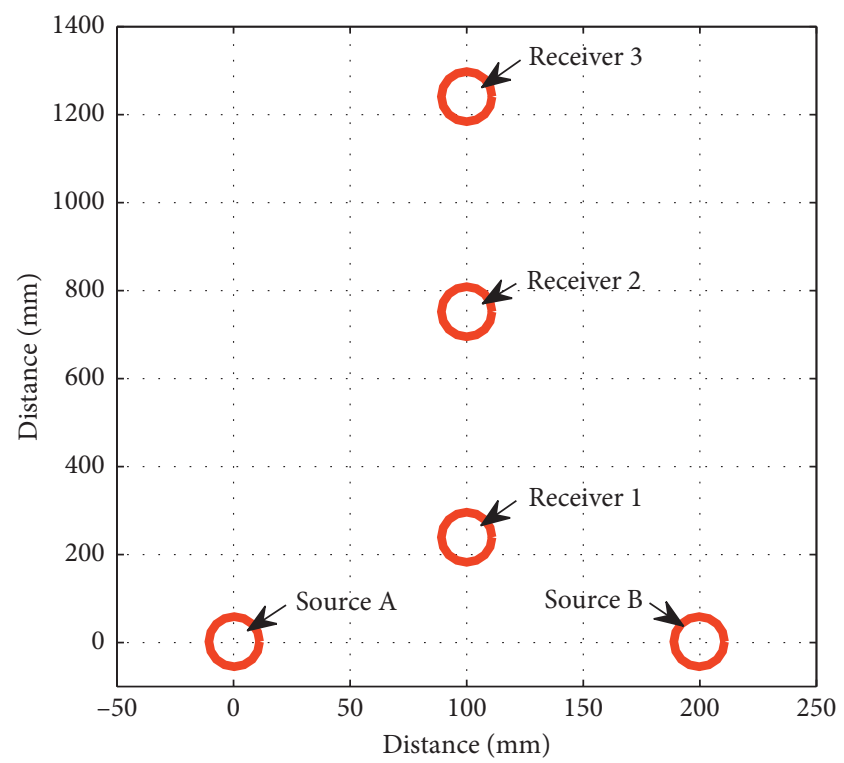

Figure 23: Positions of the different receivers and the sources in the virtual environment. 


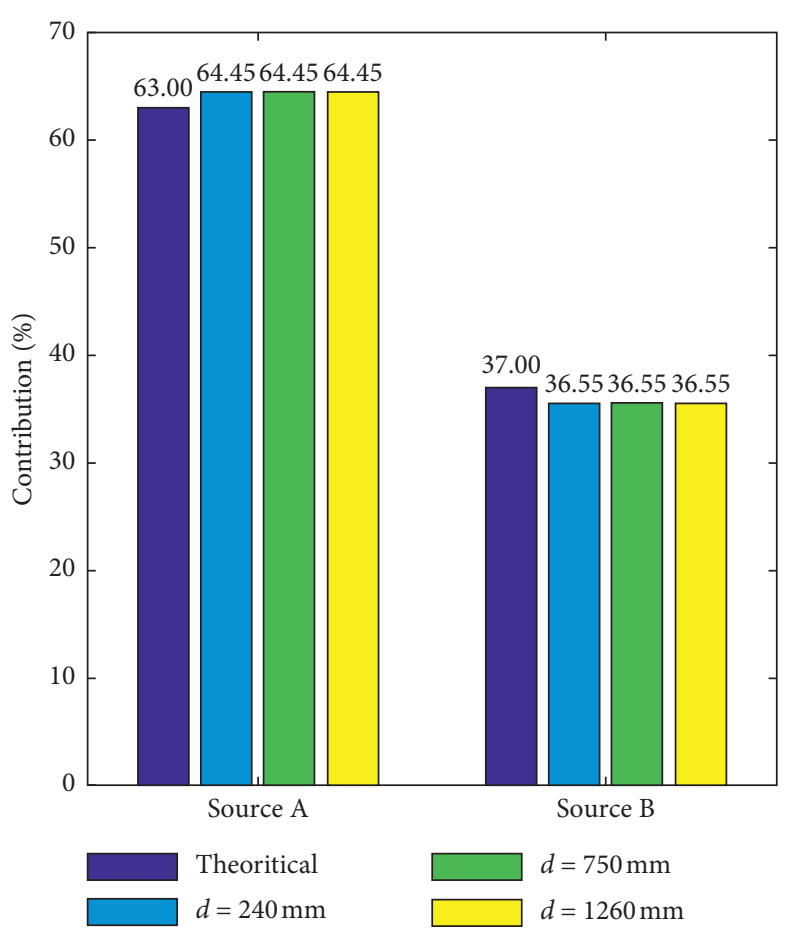

(a)

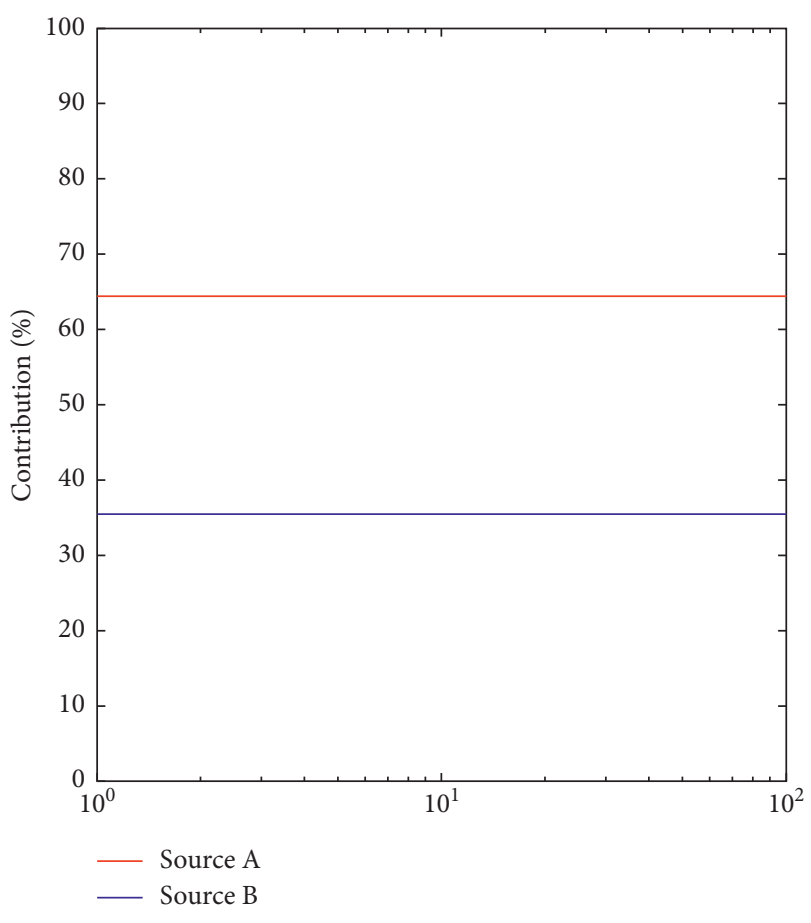

(b)

FIgURE 24: Contributions for different distances: (a) contribution per source; (b) contribution point by point.

cases, which mean that the contribution of each source for all three distances is nearly the same, and they are all equal to $64.45 \%$ and $36.55 \%$ with an error of $1.45 \%$ compared to the theoretical contribution of each source.

It can be noted that the three curves of point per point contribution are on top of each other, which further demonstrates the equality of source contributions found by the OTPA for all distances. Therefore, it can be explained by the fact that source contribution is calculated before the response synthesis and with measurements from the motors themselves. Since the speeds used are similar for all receiver distances, it is logical that the same source contributions are found. Figure 25 shows the response synthesis in function of the distance of the receiver for three distances. Two peaks can be observed at $14 \mathrm{~Hz}$ and $16 \mathrm{~Hz}$ for both the theoretical and synthesized curves, which are close to reality as the same range as the frequency of the motors studied.

Figure 26 summarizes the errors: the errors of the proposed OTPA are $2.86 \%, 3.42 \%$, and $4.01 \%$, while the errors of the traditional OTPA are $6.84 \%, 7.16 \%$, and $9.14 \%$ for $d=240 \mathrm{~mm}, d=750 \mathrm{~mm}$, and $d=1240 \mathrm{~mm}$ cases. The further the receiver from the sources, the bigger the errors. This is why the receivers should not be placed too far away from the sources. It can also be noted that the proposed OTPA is in average errors $4.28 \%$ more accurate than that of the traditional OTPA.

5.2.2. Experimental Determination of the Influence of the Distance. Three accelerometers, representing the response, are placed on top of the test bench at, respectively, $240 \mathrm{~mm}$,
$750 \mathrm{~mm}$, and $1260 \mathrm{~mm}$ from the front extremity of the cylinder. The configuration is similar to the simulation. Figure 27 presents the configuration used, and data blocks are measured at a percentage of the maximum rotational speed of the motors. The different speeds are detailed in Table 5. A minimum of $60 \%$ difference is introduced between source speeds to avoid the negative influence of correlation demonstrated in Section 5.1. Two different measurements are done to increase the amount of data to be compared.

The correlation found is displayed in Figure 28, which shows that there is a slight overestimation of source A in each measurement for every single distance. The most noticeable feature, however, is that all contributions are strictly identical no matter where the receiver is placed on the structure, which is observable in both the overall contribution per source and that point by point. In the latter, in particular, the curves corresponding to different distances overwrite one another. When a comparison test is run on those curves, a very small difference of merely $0.01 \%$ is obtained, which clearly shows that the distance in this study has nearly no influences on the source contribution results.

Figures 29 and 30 display the results of the synthesized responses at the receiver for different locations. For both measurements, a diminution of the amplitude of the harmonics in the frequency spectrum is observed as the distance between the sources and the receiver increases. The phenomenon can be explained by a damping coefficient present in the structure of the test bench. A stronger noise is also to be noted in the spectrum when the receiver is placed closer to the source, which is because the crosstalk phenomenon is discussed in Section 3.2. 


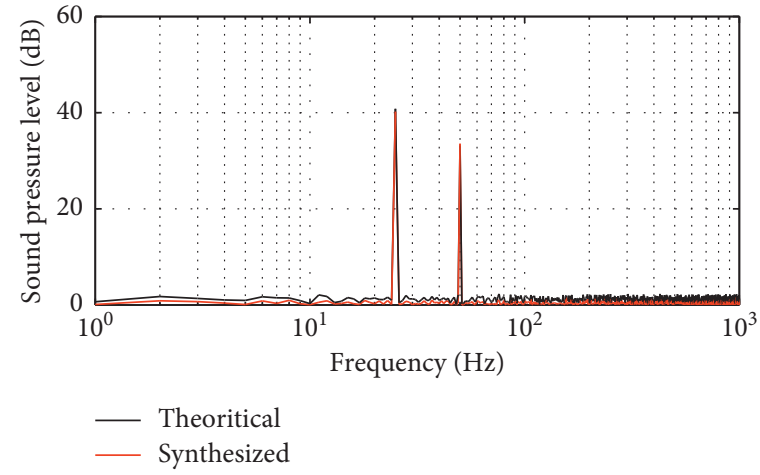

(a)

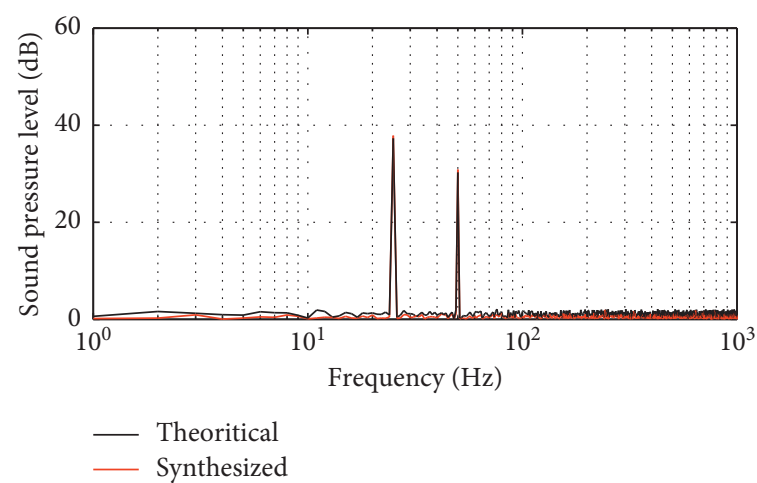

(c)

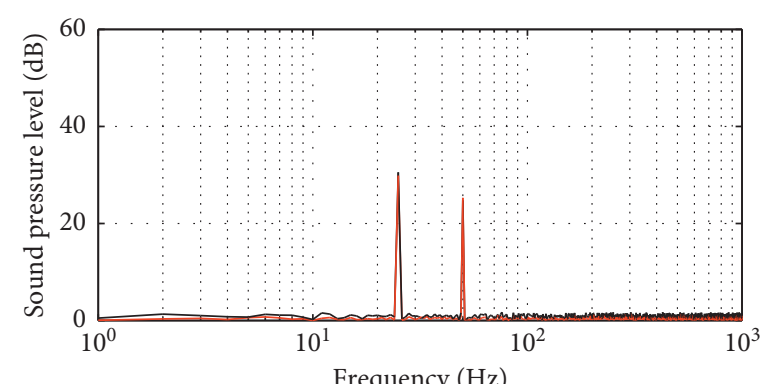

Frequency $(\mathrm{Hz})$

- Theoritical

(e)

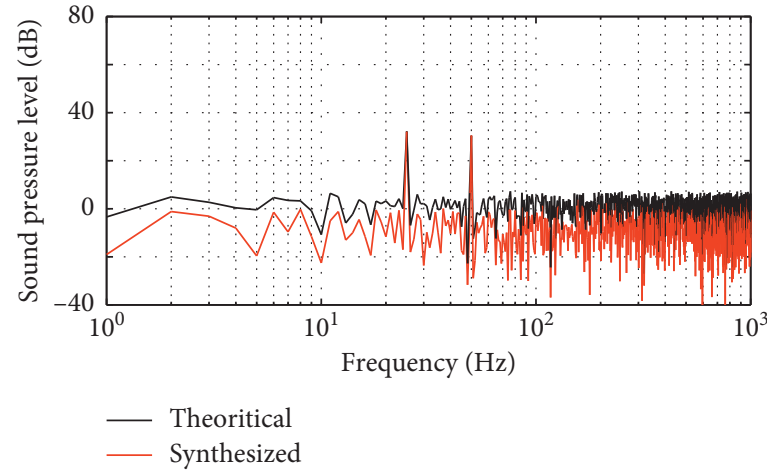

(b)

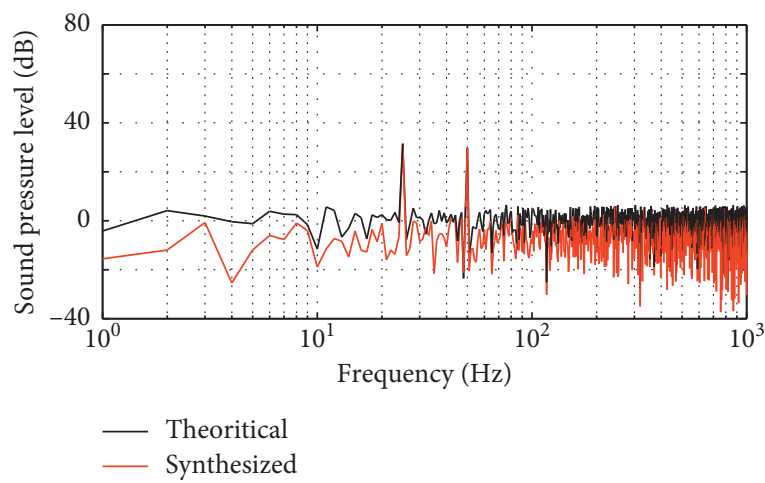

(d)

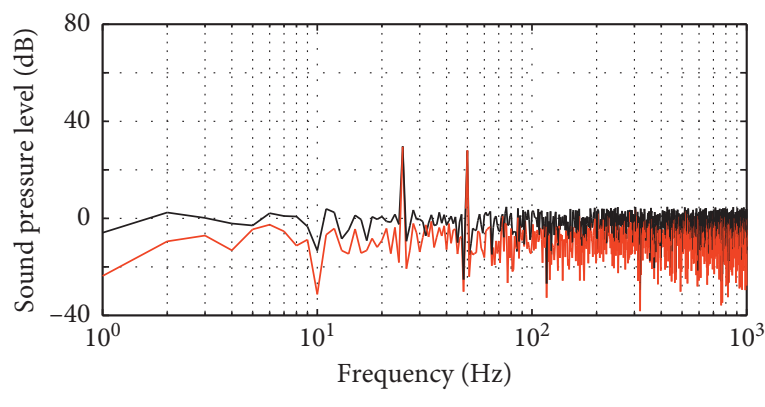

— Theoritical

— Synthesized

Figure 25: Sound pressure level spectrum and response synthesis in function of the distance: (a) $d=240 \mathrm{~mm}$; (b) $d=240 \mathrm{~mm}$; (c) $d=750 \mathrm{~mm}$; (d) $d=750 \mathrm{~mm}$; (e) $d=1400 \mathrm{~mm}$; (f) $d=1400 \mathrm{~mm}$.

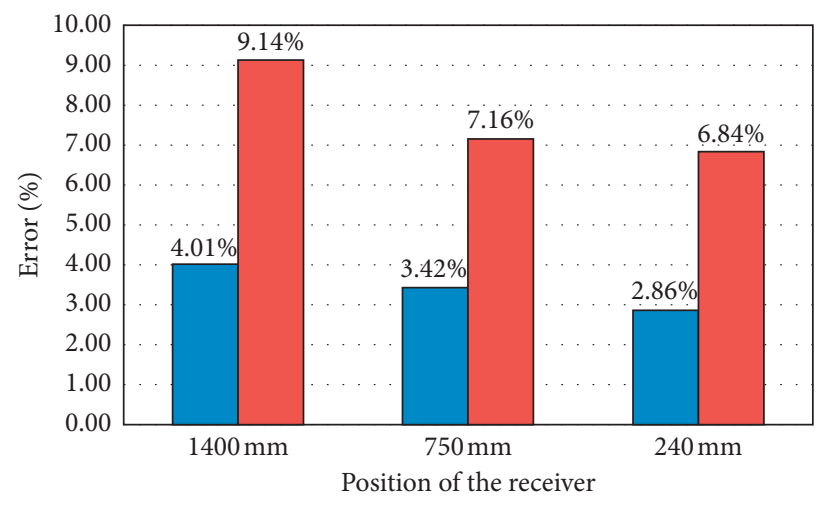

- Proposed OTPA

- Classic OTPA

FIGURE 26: Errors of the proposed and classic OTPA for different distances. 


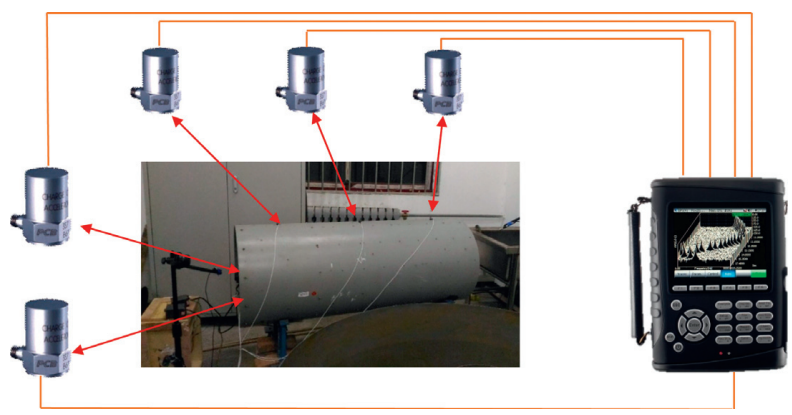

FIGURE 27: Layout of the experiment varying the receiver's distance.

TABle 5: Simulation conditions.

\begin{tabular}{|c|c|c|c|c|c|}
\hline Simulation conditions (\%) & $1(\%)$ & $2(\%)$ & $3(\%)$ & $4(\%)$ & $5(\%)$ \\
\hline Rotational speed of motor $\mathrm{A}$ & 20 & 100 & 20 & 80 & 100 \\
\hline Rotational speed of motor B & 100 & 20 & 80 & 20 & 40 \\
\hline
\end{tabular}

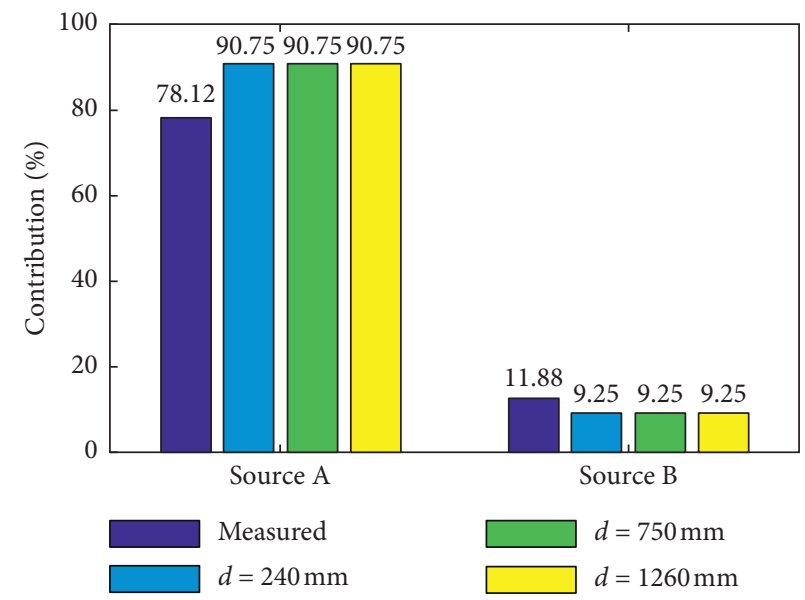

(a)

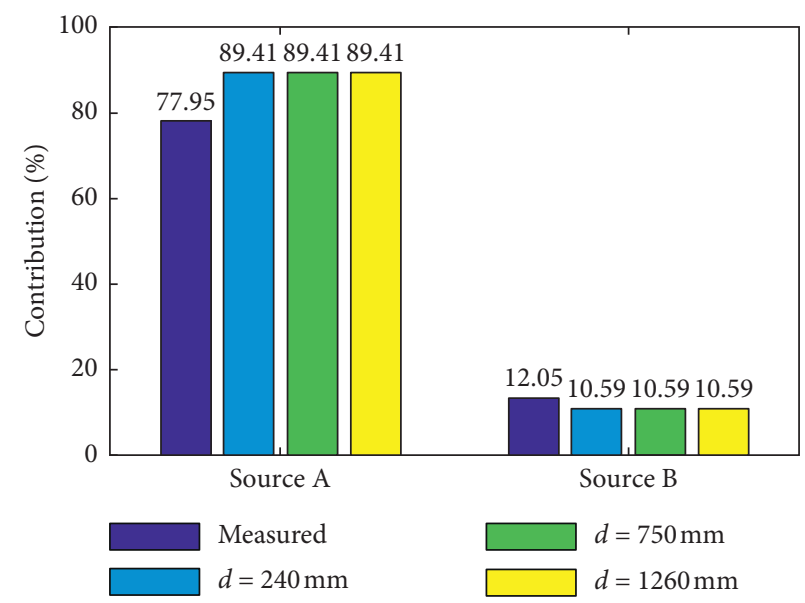

(c)

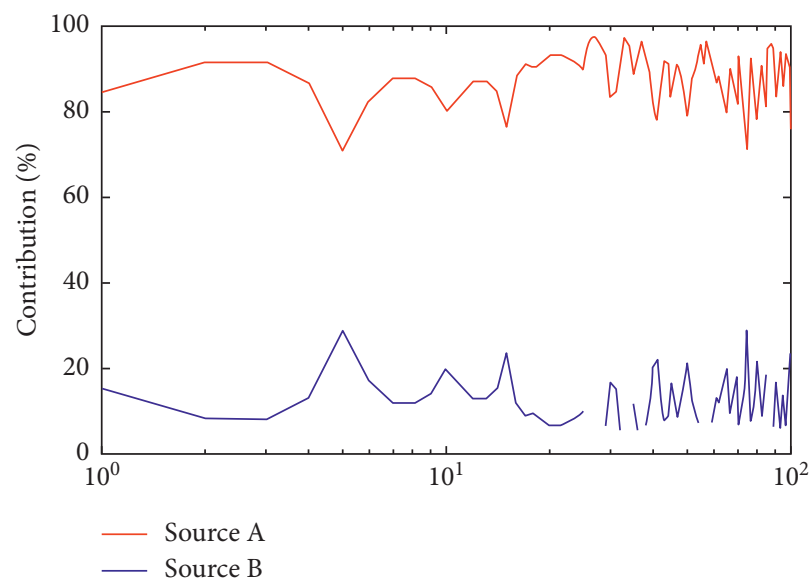

(b)

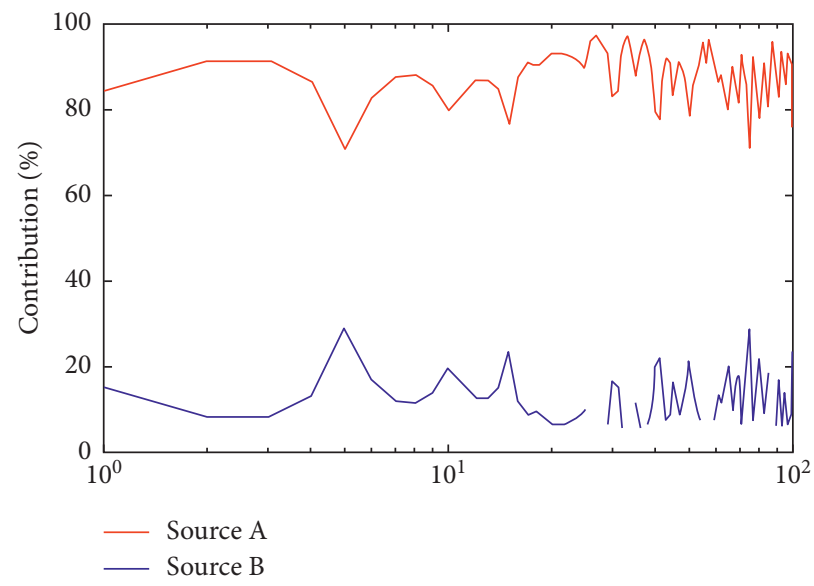

(d)

FIgURE 28: Contributions of each source for 3 different receiver distances: (a) contribution per source, measurement \#1; (b) contribution point by point, measurement \#1; (c) contribution per source, measurement \#2; (d) contribution point by point, measurement \#2. 


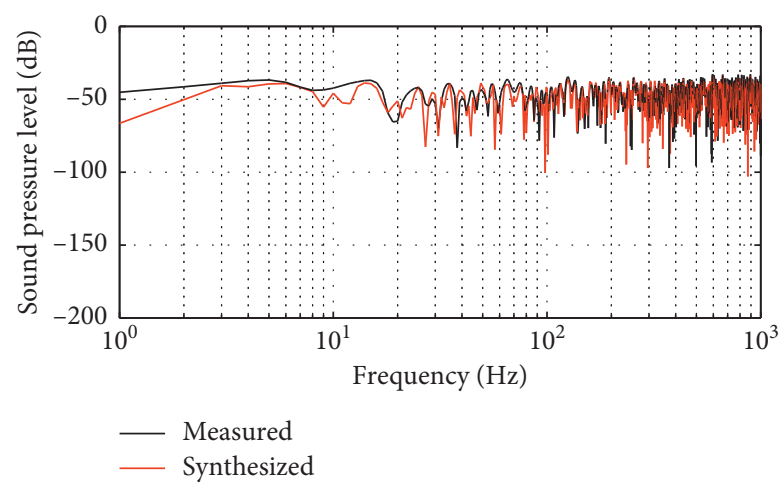

(a)

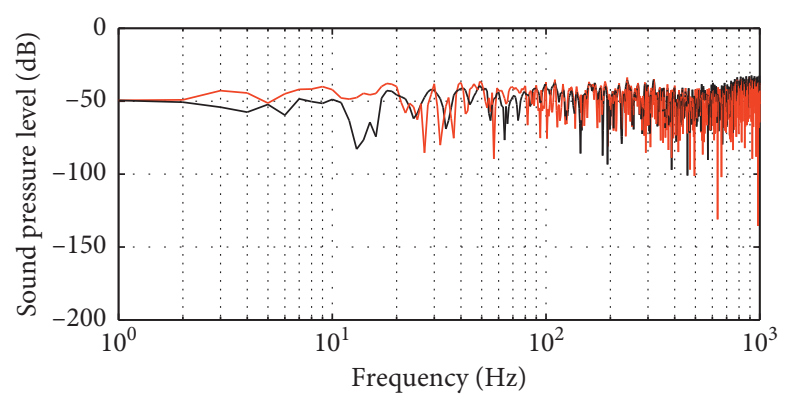

— Measured

— Synthesized

(c)

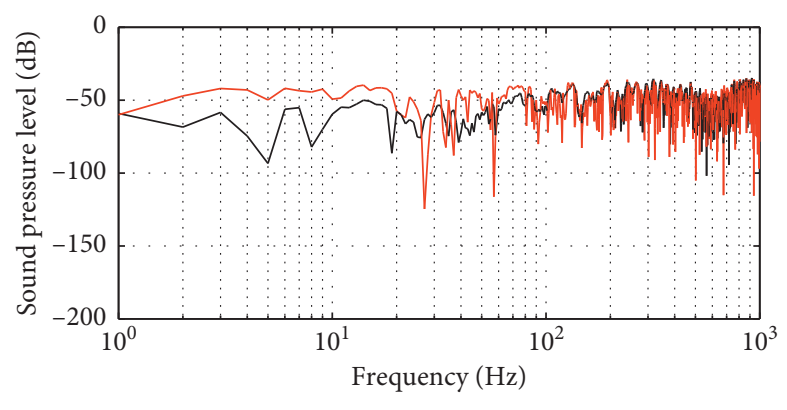

- Measured

Synthesized

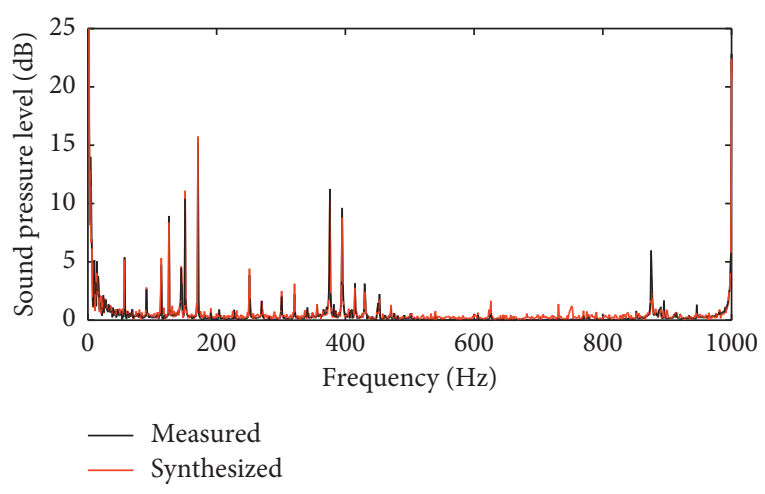

(b)

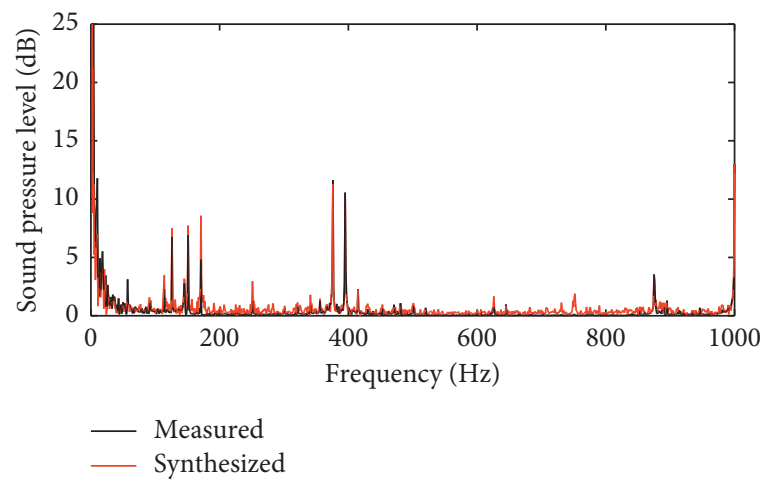

(d)

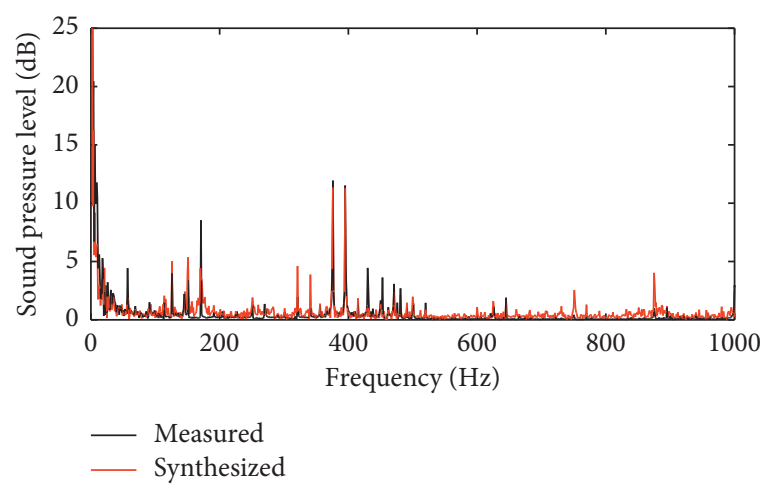

(f)

Figure 29: Response synthesis for measurement 1: (a) $d=240 \mathrm{~mm}$; (b) $d=240 \mathrm{~mm}$; (c) $d=750 \mathrm{~mm}$; (d) $d=750 \mathrm{~mm}$; (e) $d=1260 \mathrm{~mm}$; (f) $d=1260 \mathrm{~mm}$.

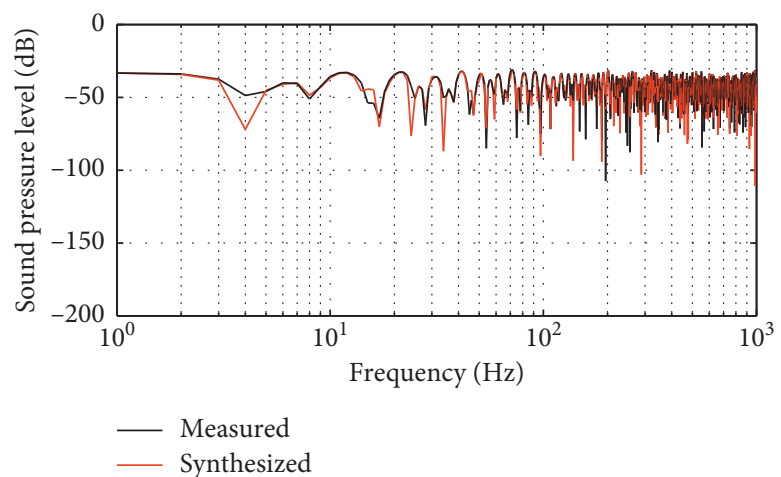

(a)

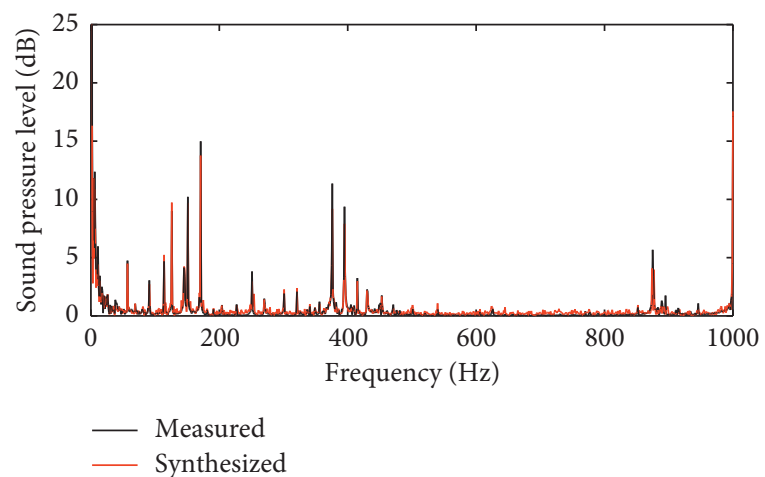

(b)

Figure 30: Continued. 


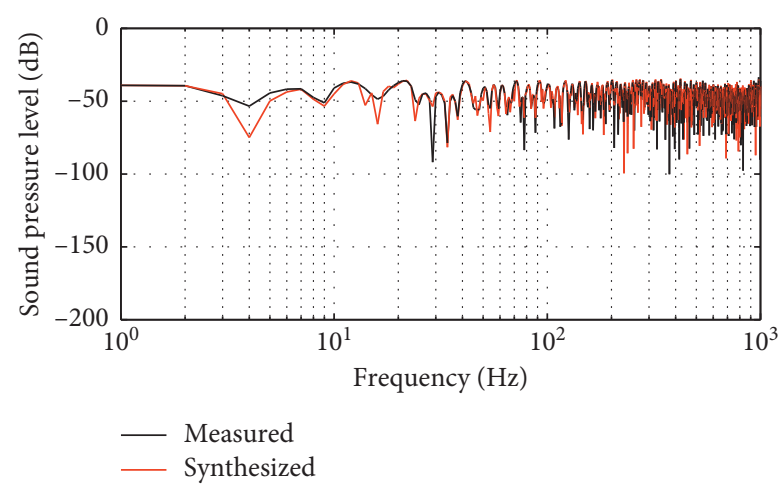

(c)

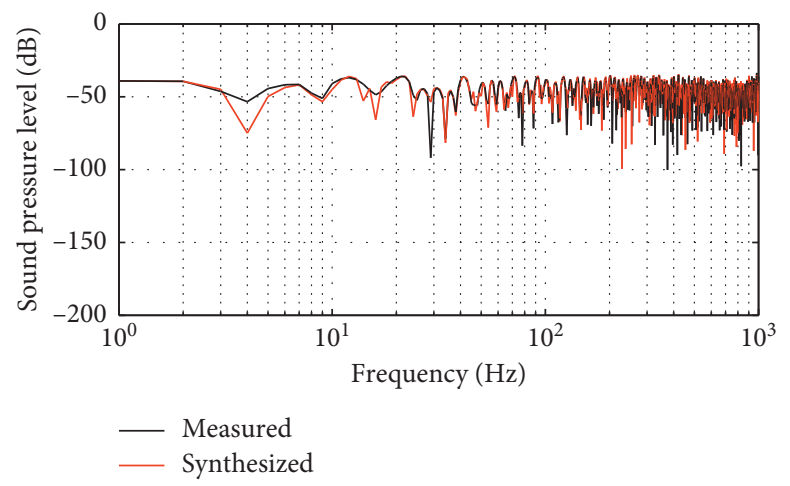

(e)

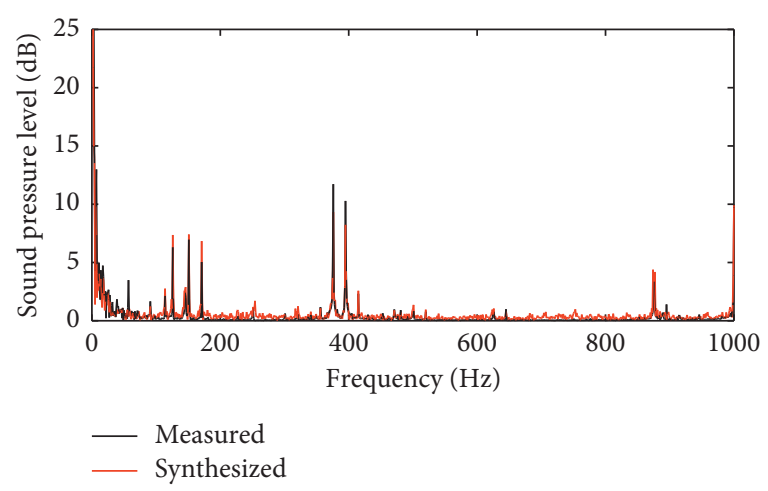

(d)

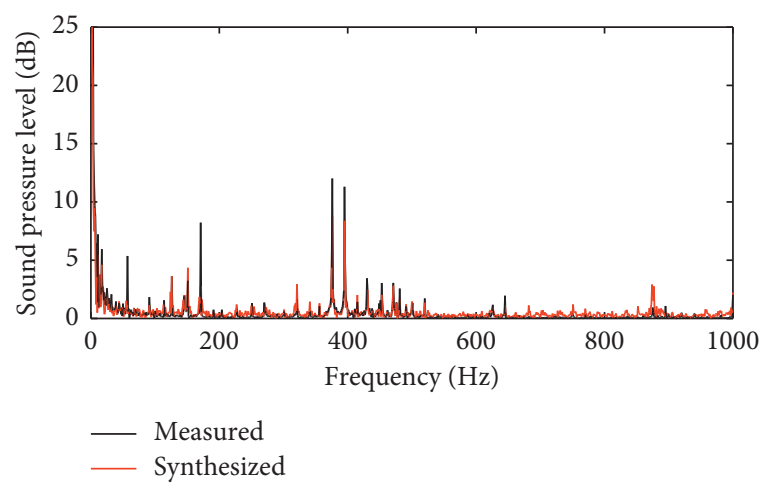

(f)

Figure 30: Response synthesis for measurement 2: (a) $d=240 \mathrm{~mm}$; (b) $d=240 \mathrm{~mm}$; (c) $d=750 \mathrm{~mm}$; (d) $d=750 \mathrm{~mm}$; (e) $d=1260 \mathrm{~mm}$; (f) $d=1260 \mathrm{~mm}$.

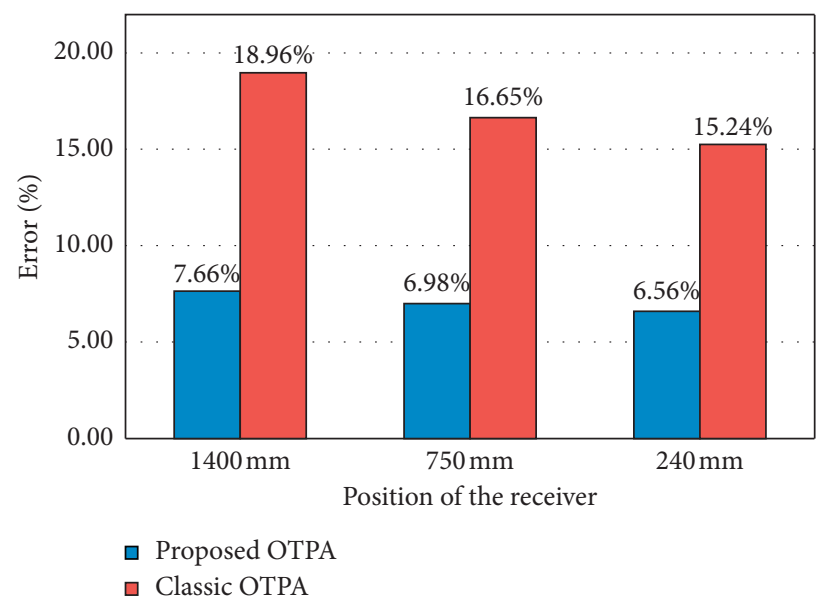

FIGURE 31: Errors of the improved and classic OTPA calculated in function of the receiver's distance.

The errors of the proposed OTPA and the traditional OTPA are shown in Figure 31: the errors of the proposed OTPA are $6.56 \%, 6.98 \%$, and $7.66 \%$, while the errors of the traditional OTPA are $15.24 \%, 16.65 \%$, and $18.96 \%$ for $d=240 \mathrm{~mm}, d=750 \mathrm{~mm}$, and $d=1400 \mathrm{~mm}$ cases, which means that there is an obvious improvement in accuracy when the receiver is closer to the sources, and the proposed OTPA also improves the accuracy by $9.88 \%$ in average.
Generally, due to crosstalk, the closer the receiver sensor is to the unrelated source, the more likely it is to be influenced and creates inaccuracies. For instance, a harmonic due to noise could be mistaken for the correct one that corresponds to the real mechanical system. However, if the receiver is too far from the sources, the amplitude may decrease too much, making it impossible to read. Furthermore, the damping also influences the transfer characteristics of mechanical systems significantly. 


\section{Conclusions}

In this paper, an effective OTPA based on SVD coupled with PCA is proposed. The performances are comparatively studied with the traditional OTPA, and main conclusions are summarized below:

(1) Crosstalk often causes OTPA path misjudgment and response synthesis inaccuracies, as the traditional OTPA only considers the least-square equation as a mean to determine the transmissibility function, while SVD- and PCA-based OTPA considers both noise and crosstalk. Therefore, the proposed OTPA can obviously eliminate path misjudgment and improve the accuracy of the contribution evaluation and response synthesis.

(2) Comparing the proposed OTPA with the traditional OTPA in the numerical, experimental, and comprehensive study, it is found that the accuracy of the response synthesis calculated with the weighted least-square equation is improved by, respectively, $4.15 \%, 4.20 \%$, and $6.24 \%$ in the correlation study and $6.71 \%$ in the distance study, which represents an average of $5.32 \%$ improvement over the traditional OTPA for all case studies.

(3) The numerical and experimental studies on a test bed with a thin cylindrical shell structure also show that the proposed OTPA with an optimum parameter $\gamma$ can effectively determine the transfer function of the mechanical system and reduce crosstalk. Therefore, the traditional OTPA can be obviously improved by the proposed OTPA.

This research is a fast theoretical and experimental basis for NVH testing and provides a more accurate OTPA for mechanical systems, which can benefit vibration source identification and vibration reduction by structural path optimization. In addition, the accurate evaluation of source contributions allows vibration monitoring and control of vibration sources to be carried out efficiently.

\section{Data Availability}

The data used to support the findings of this study are available from the corresponding author upon request.

\section{Conflicts of Interest}

The authors declare that they have no conflicts of interest.

\section{Authors' Contributions}

Wei Cheng and Diane Blamaud conceptualized, investigated, and visualized the study, helped in methodology, performed formal analysis, did data curation, and wrote the original draft. Diane Blamaud analyzed using software. Wei Cheng, Diane Blamaud, and Yapeng Chu validated the study. Wei Cheng, Yapeng Chu, and Lei Meng managed resources. Wei Cheng, Jingbai $\mathrm{Lu}$, and Basit Ali Wajid reviewed and edited the study. Wei Cheng supervised the study and administrated the project. Wei Cheng and Lei Meng were responsible for funding acquisition.

\section{Acknowledgments}

This research was funded by the Projects of National Natural Science Foundation of China (no. 51775407), the National Key Research and Development Program of China (no. 2019YFB1705403), the General Project of Joint Preresearch Fund for Equipment of Ministry of Education (no. 6141A02022121), the Innovative Scientific Program of CNNC, and the Fundamental Research Funds for the Central Universities.

\section{References}

[1] W. Cheng, Y. Lu, and Z. Zhang, "Tikhonov regularizationbased operational transfer path analysis," Mechanical Systems and Signal Processing, vol. 75, pp. 494-514, 2016.

[2] W. Cheng, Z. J. He, and Z. S. Zhang, "A comprehensive study of vibration signals for a thin shell structure using enhanced independent component analysis and experimental validation," Journal of Vibration and Acoustics-Transactions of the ASME, vol. 136, no. 4, 2014.

[3] W. Cheng, S. Lee, Z. S. Zhang, and Z. J. He, "Independent component analysis based source number estimation and its comparisons for mechanical systems," Journal of Sound and Vibration, vol. 23, no. 5, pp. 5153-5167, 2012.

[4] W. Cheng, Z. S. Zhang, S. Lee, and Z. J. He, "Source contribution evaluation of mechanical vibration signals via enhanced independent component analysis," Journal of Manufacturing Science and Engineering- Transactions of the ASME, vol. 134, no. 2, 2012.

[5] W. Cheng, Z. Zhang, S. Lee, and Z. He, "Investigations of denoising source separation technique and its application to source separation and identification of mechanical vibration signals," Journal of Vibration and Control, vol. 20, no. 14, pp. 2100-2117, 2014.

[6] G. De Sitter, C. Devriendt, P. Guillaume, and E. Pruyt, "Operational transfer path analysis," Mechanical Systems and Signal Processing, vol. 24, no. 2, pp. 416-431, 2010.

[7] L. Keersmaekers, L. Mertens, R. Penne, P. Guillaume, and G. Steenackers, "Decoupling of mechanical systems based on in-situ frequency response functions: the link-preserving, decoupling method," Mechanical Systems and Signal Processing, vol. 58-59, pp. 340-354, 2015.

[8] P. Gajdatsy, K. Janssens, W. Desmet, and H. Van der Auweraer, "Application of the transmissibility concept in transfer path analysis," Mechanical Systems and Signal Processing, vol. 24, no. 7, pp. 1963-1976, 2010.

[9] O. Guasch, C. Garcia, J. Jove, and P. Artis, "Experimental validation of the direct transmissibility approach to classical transfer path analysis on a mechanical setup," Mechanical Systems and Signal Processing, vol. 37, no. 1-2, pp. 353-369, 2013.

[10] D. de Klerk and A. Ossipov, "Operational transfer path analysis: theory, guidelines and tire noise application," $\mathrm{Me}$ chanical Systems and Signal Processing, vol. 24, no. 7, pp. 1950-1962, 2010.

[11] Y. Li and J. Weikang, "Research on the procedure for analyzing the sound quality contribution of sound sources and its application," Applied Acoustics, vol. 79, no. 75-80, pp. 75-80, 2014. 
[12] M. Reising, F. Küçükay, S. Eulert, H. Decker, and G.-F. Luehrs, "Hybrid acoustic synthesis - assessment of rear final drives in an early stage of development, on the basis of transfer path analyses and test bench measurements," Applied Acoustics, vol. 80, pp. 45-56, 2014.

[13] K. Janssens, P. Gajdatsy, L. Gielen et al., "OPAX: a new transfer path analysis method based on parametric load models," Mechanical Systems and Signal Processing, vol. 25, no. 4, pp. 1321-1338, 2011.

[14] N. B. Roozen and Q. Leclère, "On the use of artificial excitation in operational transfer path analysis," Applied Acoustics, vol. 74, no. 10, pp. 1167-1174, 2013.

[15] M. Toome, "Operational transfer path analysis, a study of source contribution predictions at low frequency," M.S. thesis, Chalmers University of Technology, Gothenburg, Sweden, 2012.

[16] R. Ström, "Operational transfer path analysis of components of a high-speed train bogie," M.S. thesis, Chalmers University of Technology, Gothenburg, Sweden, 2014.

[17] K. Noumura and J. Yoshida, "A method of transfer path analysis for vehicle interior sound with no excitation experiment," in Proceedings of the FISITA 2006 World Automotive Congress, Yokohama, Japan, October 2006. 